\title{
AN ASYMPTOTIC THEORY FOR A CLASS OF NONLINEAR ROBIN PROBLEMS. II
}

\author{
BY
}

F. A. HOWES ${ }^{1}$

\begin{abstract}
Various asymptotic phenomena exhibited by solutions of singularly perturbed Robin boundary value problems are studied in the case when the right-hand side grows faster than the square of the derivative.
\end{abstract}

1. Introduction. We consider here some extensions of our results on the nonlinear Robin problem,

$$
\begin{gathered}
\varepsilon y^{\prime \prime}=f\left(t, y, y^{\prime}\right), \quad a<t<b, \\
p_{1} y(a, \varepsilon)-p_{2} y^{\prime}(a, \varepsilon)=A, \quad q_{1} y(b, \varepsilon)+q_{2} y^{\prime}(b, \varepsilon)=B,
\end{gathered}
$$

with $f\left(t, y, y^{\prime}\right)= \pm y^{\prime 2}+h(t, y)$, published in [8]. Specifically we are interested in the existence and the asymptotic behavior (as $\varepsilon \rightarrow 0^{+}$) of solutions of the problem $(\Re)$ whose right-hand side $f$ satisfies $f\left(t, y, y^{\prime}\right)=\mathcal{O}\left(\left|y^{\prime}\right|^{n}\right)$ as $\left|y^{\prime}\right| \rightarrow \infty$ for $n>2$. Such "superquadratic" problems have been considered by the author in [9] for functions $f$ of the form $f\left(t, y, y^{\prime}\right)=h(t, y) g\left(t, y, y^{\prime}\right)$ where $g\left(t, y, y^{\prime}\right)=\vartheta\left(\left|y^{\prime}\right|^{n}\right)$, $n>2$ and $g \geqslant \nu>0$ for all $\left(t, y, y^{\prime}\right)$ of interest. However this positivity assumption on $g$ effectively eliminates the participation of nonsingular solutions of the reduced equation $f\left(t, y, y^{\prime}\right)=0$ in the asymptotic description of solutions of the problem $(\Re)$ for small values of $\varepsilon>0$. (A solution $u=u(t)$ of $f\left(t, u, u^{\prime}\right)=0$ in $[a, b]$ is said to be nonsingular if $f_{y^{\prime}}\left(t, u(t), u^{\prime}(t)\right) \neq 0$ in [a,b]; cf., for example, [10, Chapter 3].) The results of [8] for the quadratic functions $f\left(t, y, y^{\prime}\right)= \pm y^{\prime 2}+h(t, y)$ clearly show that nonsingular solutions of $f=0$ play an interesting and important role in analyzing how solutions of $(\mathcal{T})$ behave as $\varepsilon \rightarrow 0^{+}$. Thus it seems of interest to us to examine similar questions in the case that $f\left(t, y, y^{\prime}\right)=\theta\left(\left|y^{\prime}\right|^{n}\right)$ as $\left|y^{\prime}\right| \rightarrow \infty$ for $n>2$ without the restriction that $f\left(t, y, y^{\prime}\right)=h(t, y) g\left(t, y, y^{\prime}\right)$.

Such problems have not received much attention in the literature on singular perturbations apparently due to the highly nonlinear dependence of $f$ on $y^{\prime}$. The author's papers [8] and [9] contain the latest results on the problem $(\mathscr{N})$ for the functions $f$ discussed above as well as references to the work of others. Since the writing of [9], L. Perko [15] has examined turning point phenomena for problems related to $(\mathcal{T})$ using methods developed in his previous work [12], [14].

Received by the editors May 9, 1979.

AMS (MOS) subject classifications (1970). Primary 34E15; Secondary 34B15.

'Supported in part by the National Science Foundation under Grant No. MCS 78-00907 at the University of Minnesota and by the Office of Naval Research under Contract No. N00014-76-C-0326 at the University of Arizona. 
2. A first-order problem. In order to discuss the problem $(\mathcal{T})$ we will need some results on stability theory which are most clearly illustrated by a class of first-order problems. The theory discussed in this section is very straightforward and certainly not new (cf. [17, Chapter 1] or [3, Chapter 4]); however, we have not seen it expressed before in quite the exact form that we need for our purposes here.

Consider then the singularly perturbed initial value problem

$$
\varepsilon z^{\prime}=f(z), \quad a<t<b, \quad z(a, \varepsilon)=z_{0},
$$

for finite values of $a$ and $b$ and for small values of $\varepsilon>0$. If the equation $f(z)=0$ has a solution $z=\sigma$ and if $\sigma$ is stable in a sense to be made precise shortly then we anticipate that the problem $(\mathscr{F})$ has a solution $z=z(t, \varepsilon)$ such that

$$
\lim _{\varepsilon \rightarrow 0^{+}} z(t, \varepsilon)=\sigma \text { for } a<t \leqslant b .
$$

(Indeed, if $f\left(z_{0}\right)=0$ then $z(t, \varepsilon) \equiv z_{0}$ is itself a solution.) In order that the limiting relation (2.1) hold it is enough to require that either $\sigma=z_{0}$ or (if $\sigma \neq z_{0}$ )

$$
\left(\sigma-z_{0}\right) f(\lambda)>0 \text { for all } \lambda \text { in }\left(\sigma, z_{0}\right] \text {. }
$$

(Here and in what follows $(\sigma, z]=\{x: \sigma<x \leqslant z\}([\sigma, z]=\{x: \sigma \leqslant x \leqslant z\})$ if $\sigma<z$ and $(\sigma, z]=[z, \sigma)([\sigma, z]=[z, \sigma])$ if $\sigma>z$.) This follows immediately once we make the change of variable $\tau=(t-a) \varepsilon^{-1}$, rewrite $\varepsilon z^{\prime}=f(z)$ as $d z / d \tau=f(z)$, and note that condition (2.2) is just the condition for $z=\sigma$ to be an asymptotically stable rest point of the $\tau$-equation (cf. [6, Chapter 3]).

Our result on $(\mathscr{F})$ is contained in the following lemma.

Lemma 2.1. Assume that the equation $f(z)=0$ has a solution $z=\sigma$ and that the function $f$ is continuously differentiable in $\left[\sigma, z_{0}\right]$. Then for all values of $z_{0}$ such that $z_{0}=\sigma$ or $\left(\right.$ if $\left.z_{0} \neq \sigma\right)\left(\sigma-z_{0}\right) f(\lambda)>0$ for all $\lambda$ in $\left(\sigma, z_{0}\right]$ the problem $(\mathcal{F})$ has $a$ solution $z=z(t, \varepsilon)$ for each sufficiently small $\varepsilon>0$. Moreover, for $t$ in $[a, b]$ we have that $z(t, \varepsilon)=\sigma+w_{L}(t, \varepsilon)$, where $w_{L}(a, \varepsilon)=z_{0}-\sigma$ and $\lim _{\varepsilon \rightarrow 0^{+}} w_{L}(t, \varepsilon)=0$ for $a<t \leqslant b$.

A result analogous to Lemma 2.1 is valid if the problem $(\mathscr{F})$ is replaced by the problem

$$
\varepsilon z^{\prime}=f(z), \quad a<t<b, \quad z(b, \varepsilon)=z_{1} .
$$

This follows after replacing $t$ by $a+b-t$ in (G) and applying Lemma 2.1 to the transformed problem. We leave its precise formulation to the reader, except to note that the inequality corresponding to (2.2) becomes $\left(\sigma-z_{1}\right) f(\lambda)<0$ for all $\lambda$ in $\left(\sigma, z_{1}\right]$ (if $\left.z_{1} \neq \sigma\right)$.

The results of this section have a direct connection with a special class of Robin problems of the form $(\mathfrak{T})$ to which we now turn.

3. Some special problems. We begin our discussion of the problem $(\mathcal{T})$ by first considering a particularly simple problem, namely

$$
\begin{aligned}
\varepsilon y^{\prime \prime}=f\left(y^{\prime}\right), & a<t<b, \\
p_{1} y(a, \varepsilon)-p_{2} y^{\prime}(a, \varepsilon)=A, & q_{1} y(b, \varepsilon)+q_{2} y^{\prime}(b, \varepsilon)=B .
\end{aligned}
$$


Here the constants $p_{1}, p_{2}, q_{1}$ and $q_{2}$ are nonnegative with $p_{1}+q_{1}>0$ and $p_{2}+q_{2}>0$, and $f(z)=\mathcal{\theta}\left(|z|^{n}\right)$ as $|z| \rightarrow \infty$ for $n>2$. The results we obtain for solutions of $\left(\Re_{1}\right)$ will turn out to be characteristic for most solutions of the general problem $(\Re)$.

Suppose first that $p_{1}=0$ and $p_{2}=1$. We consider then the prblem

$$
\begin{gathered}
\varepsilon y^{\prime \prime}=f\left(y^{\prime}\right), \quad a<t<b, \\
-y^{\prime}(a, \varepsilon)=A, \quad q_{1} y(b, \varepsilon)+q_{2} y^{\prime}(b, \varepsilon)=B .
\end{gathered}
$$

After setting $z=y^{\prime}$ and disregarding (for the moment) the boundary condition at $t=b$ we see that the problem $\left(\mathscr{N}_{2}\right)$ is precisely the initial value problem $(\mathscr{F})$ of the previous section with $z_{0}=-A$. Now solutions of $(\mathscr{F})$ are described throughout $[a, b]$ by the stable zeros of the function $f$ with the possible exception of a small neighborhood of the point $t=a$ (cf. Lemma 2.1). Returning to the problem $\left(\Re_{2}\right)$ we expect that if a stable solution $u$ of $f\left(u^{\prime}\right)=0$ also satisfies the right-hand boundary condition, that is, if $q_{1} u(b)+q_{2} u^{\prime}(b)=B$, then the solution of $\left(\mathscr{T}_{2}\right)$ for small $\varepsilon>0$ is represented throughout $[a, b]$ by this function $u$. This leads us to consider the so-called reduced problem

$$
f\left(u^{\prime}\right)=0, \quad a<t<b, \quad q_{1} u(b)+q_{2} u^{\prime}(b)=B,
$$

and to seek solutions of $\left(\Re_{R}\right)$ which are stable in the sense described in Lemma 2.1 . The solutions of $f\left(u^{\prime}\right)=0$ are clearly straight lines of slope $\sigma$ where $f(\sigma)=0$ and therefore the solution of $\left(\Re_{R}\right)$ is $u=u_{R}(t)=\sigma t+c$ where

$$
c=q_{1}^{-1}\left[B-\sigma\left(q_{1} b+q_{2}\right)\right] \text {. }
$$

(Note that $q_{1}>0$ by our above assumptions since $p_{1}=0$.)

We can now state and prove an existence and estimation result for the problem $\left(\Re_{2}\right)$.

THEOREM 3.1. Assume that the reduced problem $\left(\mathscr{R}_{R}\right)$ has a solution $u=u_{R}(t)=$ $\sigma_{R} t+c$ and that the function $f$ is continuously differentiable in $\left[\sigma_{R},-A\right]$. Assume also that either $\sigma_{R}=-A$ or $\left(\right.$ if $\left.\sigma_{R} \neq-A\right)\left(\sigma_{R}+A\right) f(\lambda)>0$ for all $\lambda$ in $\left(\sigma_{R},-A\right]$. Then there exists an $\varepsilon_{0}>0$ such that the problem $\left(\mathscr{T}_{2}\right)$ has a unique solution $y=y(t, \varepsilon)$ whenever $0<\varepsilon \leqslant \varepsilon_{0}$. In addition, for $t$ in $[a, b]$ we have that

$$
y(t, \varepsilon)=u_{R}(t)+\mathcal{O}\left(w_{L}(t, \varepsilon)\right) \text { and } y^{\prime}(t, \varepsilon)=\sigma_{R}+\vartheta\left(w_{L}^{\prime}(t, \varepsilon)\right),
$$

where the function $w_{L}$ is a solution of $\varepsilon w_{L}^{\prime \prime}=f\left(\sigma_{R}+w_{L}^{\prime}\right), a<t<b, w_{L}^{\prime}(a, \varepsilon)=$ $-\left(\sigma_{R}+A\right)$, satisfying $\lim _{\varepsilon \rightarrow 0^{+}} w_{L}(t, \varepsilon)=0$ for $a \leqslant t \leqslant b$ and $\lim _{\varepsilon \rightarrow 0^{+}} w_{L}^{\prime}(t, \varepsilon)=0$ for $a<t \leqslant b$.

Proof. The uniqueness of $y$ follows immediately from the maximum principle (cf. [16]). To prove the existence of a solution satisfying the limiting relations (3.1) we assume without loss of generality that $\sigma_{R}=0$ (and so $u_{R}(t) \equiv c=q_{1}^{-1} B$ ). If $A=0$ then $y(t, \varepsilon) \equiv 0$ (and $w_{L} \equiv 0$ ). Thus suppose that $A \neq 0$. The existence of a function $w_{L}$ with the above properties follows from our stability assumption (cf. §2) if $\varepsilon$ is sufficiently small, say $0<\varepsilon \leqslant \varepsilon_{0}$. In addition, if $-A<0$ then $w_{L}>0$ and if $-A>0$ then $w_{L}<0$. 
Define now for $t$ in $[a, b]$ and $0<\varepsilon \leqslant \varepsilon_{0}$,

$$
\left.\begin{array}{l}
\alpha(t, \varepsilon)=c+w_{L}(t, \varepsilon) \\
\beta(t, \varepsilon) \equiv c
\end{array}\right\} \quad \text { if }-A>0,
$$

and

$$
\left.\begin{array}{l}
\alpha(t, \varepsilon) \equiv c \\
\beta(t, \varepsilon)=c+w_{L}(t, \varepsilon)
\end{array}\right\} \quad \text { if }-A<0 .
$$

We consider just the case $-A<0$ since the case $-A>0$ is handled similarly. It is clear that $-\alpha^{\prime}(a, \varepsilon) \leqslant A \leqslant-\beta^{\prime}(a, \varepsilon), q_{1} \alpha(b, \varepsilon)+q_{2} \alpha^{\prime}(b, \varepsilon) \leqslant B \leqslant q_{1} \beta(b, \varepsilon)+$ $q_{2} \beta^{\prime}(b, \varepsilon)$ and that $\varepsilon \alpha^{\prime \prime} \geqslant f\left(\alpha^{\prime}\right)$ and $\varepsilon \beta^{\prime \prime} \leqslant f\left(\beta^{\prime}\right)$ for $t$ in $(a, b)$ and $0<\varepsilon \leqslant \varepsilon_{0}$. If we could conclude that the problem $\left(\Re_{2}\right)$ had a solution $y=y(t, \varepsilon)$ satisfying $\alpha(t, \varepsilon)$ $\leqslant y(t, \varepsilon) \leqslant \beta(t, \varepsilon)$ for $t$ in $[a, b]$ and $0<\varepsilon \leqslant \varepsilon_{0}$ then the theorem would be proved. However such a conclusion cannot be drawn immediately here since $f\left(y^{\prime}\right)=$ $\vartheta\left(\left|y^{\prime}\right|^{n}\right)$ as $\left|y^{\prime}\right| \rightarrow \infty$ for $n>2$ (cf. [11]). What is required (cf. Heidel's theorem in [7] or [9]) is an a priori bound on the derivative of any solution $y$ of $\varepsilon y^{\prime \prime}=f\left(y^{\prime}\right)$, $a<t<b$, satisfying $\alpha(t, \varepsilon) \leqslant y(t, \varepsilon) \leqslant \beta(t, \varepsilon)$. It will turn out (not surprisingly) that

$$
-A \leqslant y^{\prime}(t, \varepsilon) \leqslant 0 \text { for } a \leqslant t \leqslant b,
$$

and therefore the conclusion of Theorem 3.1 follows from Heidel's theorem. To verify (3.2) (and at the same time obtain a sharper estimate for $y^{\prime}(t, \varepsilon)$ ) note first that $y^{\prime}(t, \varepsilon) \leqslant 0$ by the maximum principle (cf. [16] or [2, §2]). In calculating a lower bound on $y^{\prime}$ we proceed indirectly by noting that for $\alpha \leqslant y \leqslant \beta, y$ is a solution of the following Dirichlet problem in $\left(t_{1}, t_{2}\right) \subset(a, b)$.

$$
\begin{array}{cl}
\varepsilon y^{\prime \prime}=f\left(y^{\prime}\right), & t_{1}<t<t_{2}, \\
y\left(t_{1}, \varepsilon\right)=c+\eta\left(t_{1}, \varepsilon\right), & y\left(t_{2}, \varepsilon\right)=c+\eta\left(t_{2}, \varepsilon\right),
\end{array}
$$

where the positive function $\eta$ is of order $\theta\left(w_{L}(t, \varepsilon)\right)$ and $\eta\left(t_{1}, \varepsilon\right) \geqslant \eta\left(t_{2}, \varepsilon\right)$. Fix $t_{0}$ in $(a, b]$ and let $t_{1}=t_{0}-\delta_{1}$ and $t_{2}=t_{0}$ for a small positive constant $\delta_{1}$. Define now for $t$ in $\left[t_{1}, t_{2}\right]$ and $0<\varepsilon \leqslant \varepsilon_{0}$,

$$
\alpha_{1}(t, \varepsilon)=c+\eta\left(t_{2}, \varepsilon\right)-\mu\left(t_{0}-t\right), \quad \beta_{1}(t, \varepsilon)=c+\eta\left(t_{2}, \varepsilon\right)+\mu\left(t_{0}-t\right),
$$

where $\mu=\mu(\varepsilon)=\delta_{1}^{-1}\left(\eta\left(t_{0}-\delta_{1}, \varepsilon\right)-\eta\left(t_{0}, \varepsilon\right)\right)$ is positive and of order $\theta\left(w_{L}^{\prime}\left(t_{0}, \varepsilon\right)\right)$. Clearly $\alpha_{1}\left(t_{j}, \varepsilon\right) \leqslant y\left(t_{j}, \varepsilon\right) \leqslant \beta_{1}\left(t_{j}, \varepsilon\right)$ for $j=1,2$ and we just have to show that $\varepsilon \alpha_{1}^{\prime \prime} \geqslant f\left(\alpha_{1}^{\prime}\right)$ and $\varepsilon \beta_{1}^{\prime \prime} \leqslant f\left(\beta_{1}^{\prime}\right)$, that is, $f\left(\alpha_{1}^{\prime}\right) \leqslant 0 \leqslant f\left(\beta_{1}^{\prime}\right)$. However these inequalities follow directly from our stability assumption for $f\left(\alpha_{1}^{\prime}\right)=f(\mu)<0<f(-\mu)=f\left(\beta_{1}^{\prime}\right)$ since $\mu>0$. Therefore the function $y$ (which is a solution of the problem $(\mathcal{H}$ ) with $t_{1}=t_{0}-\delta_{1}$ and $\left.t_{2}=t_{0}\right)$ satisfies $\alpha_{1} \leqslant y \leqslant \beta_{1}$, that is, $\left|y(t, \varepsilon)-c_{1}\right| \leqslant \mu\left(t_{0}-t\right)$ for $t_{0}-\delta_{1} \leqslant t \leqslant t_{0}$ and $c_{1}=c+\eta\left(t_{0}, \varepsilon\right)$. We conclude directly that $\left|y^{\prime}\left(t_{0}^{-}, \varepsilon\right)\right| \leqslant$ $\mu$, so that in particular $\left|y^{\prime}\left(t_{0}, \varepsilon\right)\right| \leqslant \mu$. Thus for each $t$ in $(a, b],\left|y^{\prime}(t, \varepsilon)\right| \leqslant \mu(t, \varepsilon)$ where $\mu(t, \varepsilon)=\vartheta\left(\left|w_{L}^{\prime}(t, \varepsilon)\right|\right)$. Finally we have that $y^{\prime}(a, \varepsilon)>-A$ since $c \leqslant y(t, \varepsilon)$ $\leqslant c+w_{L}(t, \varepsilon)$ in $[a, b]$. This concludes the proof of Theorem 3.1.

The existence of the function $w_{L}=w_{L}(t, \varepsilon)$ appearing in the conclusion of Theorem 3.1 is assured by our assumptions; however, we are more interested in its asymptotic behavior as $\varepsilon \rightarrow 0^{+}$. To this end we can often estimate $w_{L}$ quite closely 
by replacing $f$ with say a polynomial approximation and solving the resulting problem in closed form. The most obvious example of this is the linear approximation (cf. Remark 3.2) but higher order comparison problems are usually required owing to the nonlinear dependence of $f$ on $y^{\prime}$. For this reason we have presented the error term in (3.1) in this general form.

We consider now the problem $\left(\mathscr{T}_{1}\right)$ under the assumption that $p_{1} \geqslant 0$ and $p_{2}>0$. As with the problem $\left(\Re_{2}\right)$ we assume that the associated reduced problem $\left(\Re_{R}\right)$ has a solution $u=u_{R}(t)$. If $u_{R}$ is stable in a sense analogous to that described in Theorem 3.1 we expect that the problem $\left(\Re_{1}\right)$ has a solution $y=y(t, \varepsilon)$ which is close to $u_{R}$ in $[a, b]$. The precise result is the next theorem.

THEOREM 3.2. Assume that the reduced problem $\left(\Re_{R}\right)$ has a solution

$$
u=u_{R}(t)=\sigma_{R} t+c
$$

and that the function $f$ is continuously differentiable in $\left[\sigma_{R}, p_{2}^{-1}\left(p_{1} u_{R}(a)-A\right)\right]$. Assume also that either $p_{1} u_{R}(a)-p_{2} \sigma_{R}=A$ or $\left(\right.$ if $\left.p_{1} u_{R}(a)-p_{2} \sigma_{R} \neq A\right)\left(p_{1} u_{R}(a)-\right.$ $\left.p_{2} \sigma_{R}-A\right) f(\lambda)<0$ for all $\lambda$ in $\left(\sigma_{R}, p_{2}^{-1}\left(p_{1} u_{R}(a)-A\right)\right]$. Then the conclusion of Theorem 3.1 is valid with the exception that the function $w_{L}$ satisfies $w_{L}^{\prime}(a, \varepsilon)=$ $p_{2}^{-1}\left(p_{1} u_{R}(a)-p_{2} \sigma_{R}-A\right)$ instead of $w_{L}^{\prime}(a, \varepsilon)=-\left(\sigma_{R}+A\right)$.

Proof. This theorem is proved in exactly the same manner as Theorem 3.1. After normalizing so that $\sigma_{R}=0$ simply define, for $a \leqslant t \leqslant b$ and $\varepsilon>0$ sufficiently small,

$$
\left.\begin{array}{l}
\alpha(t, \varepsilon) \equiv c \\
\beta(t, \varepsilon)=c+w_{L}(t, \varepsilon)
\end{array}\right\} \quad \text { if } p_{1} u_{R}(a)<A,
$$

and

$$
\left.\begin{array}{l}
\alpha(t, \varepsilon)=c+w_{L}(t, \varepsilon) \\
\beta(t, \varepsilon) \equiv c
\end{array}\right\} \quad \text { if } p_{1} u_{R}(a)>A,
$$

and proceed as before.

The basic assumption in the two previous theorems was the existence of a stable solution $u$ of the reduced equation $f\left(u^{\prime}\right)=0$ which satisfied the right-hand boundary condition. We could just as well have assumed that $u$ satisfied the left-hand boundary condition and then proceeded to impose stability conditions on it so that the result corresponding to Theorem 3.2 was valid. The appropriate reduced problem is then

$$
f\left(u^{\prime}\right)=0, \quad a<t<b, \quad p_{1} u(a)-p_{2} u^{\prime}(a)=A,
$$

and the expected result follows by making the change of variable $t \rightarrow a+b-t$ and applying Theorem 3.2 to the transformed problem. (Note that we now require $q_{1} \geqslant 0$ and $q_{2}>0$.) We leave its formulation to the reader.

Up to now we have considered how solutions of the problem $\left(\mathscr{N}_{1}\right)$ can exhibit nonuniform behavior at $t=a$ or $t=b$ (that is, boundary layer behavior). Suppose though that the following situation presents itself. The reduced problems $\left(\Re_{L}\right)$ and $\left(\Re_{R}\right)$ have solutions $u=u_{L}(t)=\sigma_{L} t+c$ and $u=u_{R}(t)=\sigma_{R} t+c^{\prime}\left(\sigma_{L} \neq \sigma_{R}\right)$ 
which intersect at a point $t_{0}$ in $(a, b)$, that is, $u_{L}\left(t_{0}\right)=u_{R}\left(t_{0}\right)$ and $u_{L}^{\prime}\left(t_{0}\right) \neq u_{R}^{\prime}\left(t_{0}\right)$. If these solutions are stable in the sense that $f^{\prime}\left(\sigma_{L}\right) \geqslant 0$ and $f^{\prime}\left(\sigma_{R}\right) \leqslant 0$ it is reasonable to ask under what additional conditions there exists a solution $y=y(t, \varepsilon)$ of the problem $\left(\mathscr{T}_{1}\right)$ which converges to the "angular" path $u_{1}(t)$ defined by $u_{1}(t)=u_{L}(t)$ for $a \leqslant t \leqslant t_{0}$ and $u_{1}(t)=u_{R}(t)$ for $t_{0} \leqslant t \leqslant b$. Indeed, this question was answered many years ago by Haber and Levinson [5] for the Dirichlet problem $(\mathcal{T})$ (that is, $p_{1}=q_{1}=1$ and $\left.p_{2}=q_{2}=0\right)$. Their result for the simpler Dirichlet problem $\left(\Re_{1}\right)$ is that if the corresponding reduced problems $\left(\mathscr{R}_{L}\right)$ and $\left(\Re_{R}\right)$ have such stable intersecting solutions $u_{L}$ and $u_{R}$ then the problem $\left(\Re_{1}\right)$ has a solution $y=y(t, \varepsilon)$ for each sufficiently small $\varepsilon>0$ such that $\lim _{\varepsilon \rightarrow 0^{+}} y(t, \varepsilon)=u_{1}(t)$ for $a \leqslant t \leqslant b$ and

$$
\lim _{\varepsilon \rightarrow 0^{+}} y^{\prime}(t, \varepsilon)= \begin{cases}\sigma_{L} & \text { for } a \leqslant t<t_{0}, \\ \sigma_{R} & \text { for } t_{0}<t \leqslant b,\end{cases}
$$

provided $\left(\sigma_{R}-\sigma_{L}\right) f(\lambda)>0$ for all $\lambda$ in $\left(\sigma_{L}, \sigma_{R}\right)$.

It is possible to state an analogous result for the Robin problem $\left(\Re_{1}\right)$ under the additional assumption that $p_{1}>0$ and $q_{1}>0$. This is the content of the next theorem.

THEOREM 3.3. Assume that the reduced problems $\left(\Re_{L}\right)$ and $\left(\Re_{R}\right)$ have solutions $u=u_{L}(t)=\sigma_{L} t+c$ and $u=u_{R}(t)=\sigma_{R} t+c^{\prime}\left(\sigma_{L} \neq \sigma_{R}\right)$ which intersect at a point $t_{0}$ in $(a, b)$. Assume also that the function $f$ is continuously differentiable in $\left[\sigma_{L}, \sigma_{R}\right]$ and that $\left(\sigma_{R}-\sigma_{L}\right) f(\lambda)>0$ for all $\lambda$ in $\left(\sigma_{L}, \sigma_{R}\right)$. Then there exists an $\varepsilon_{0}>0$ such that the problem $\left(\mathcal{R}_{1}\right)$ with $p_{1}>0$ and $q_{1}>0$ has a unique solution $y=y(t, \varepsilon)$ whenever $0<\varepsilon \leqslant \varepsilon_{0}$. In addition, we have that

$$
\begin{array}{cc}
y(t, \varepsilon)=u_{1}(t)+\mathcal{O}(w(t, \varepsilon)) & \text { for } a \leqslant t \leqslant b, \\
y^{\prime}(t, \varepsilon)=\sigma_{L}+\vartheta\left(w^{\prime}(t, \varepsilon)\right) & \text { for } a \leqslant t \leqslant t_{0},
\end{array}
$$

and

$$
y^{\prime}(t, \varepsilon)=\sigma_{R}+\vartheta\left(w^{\prime}(t, \varepsilon)\right) \text { for } t_{0} \leqslant t \leqslant b
$$

Here the continuous function $w$ is a solution of

$$
\begin{array}{lll}
\varepsilon w^{\prime \prime}=f\left(\sigma_{L}+w^{\prime}\right), & a<t<t_{0}, & w^{\prime}\left(t_{0}^{-}, \varepsilon\right)=\frac{1}{2}\left(\sigma_{R}-\sigma_{L}\right), \\
\varepsilon w^{\prime \prime}=f\left(\sigma_{R}+w^{\prime}\right), & t_{0}<t<b, & w^{\prime}\left(t_{0}^{+}, \varepsilon\right)=\frac{1}{2}\left(\sigma_{L}-\sigma_{R}\right),
\end{array}
$$

satisfying $\lim _{\varepsilon \rightarrow 0^{+}} w(t, \varepsilon)=0$ for $a \leqslant t \leqslant b$ and $\lim _{\varepsilon \rightarrow 0^{+}} w^{\prime}(t, \varepsilon)=0$ for $a \leqslant t<t_{0}$ and $t_{0}<t \leqslant b$.

Proof. This theorem is proved in essentially the same manner as Theorem 3.1. The bounding functions $\alpha$ and $\beta$ are defined as follows.

(i) If $\sigma_{L}<\sigma_{R}$ then $\alpha(t, \varepsilon)=u_{1}(t), a \leqslant t \leqslant b$, and

$$
\beta(t, \varepsilon)= \begin{cases}u_{L}(t)+w(t, \varepsilon)+p_{2} p_{1}^{-1} w^{\prime}(a, \varepsilon), & a \leqslant t \leqslant t_{0} \\ u_{R}(t)+w(t, \varepsilon)-q_{2} q_{1}^{-1} w^{\prime}(b, \varepsilon), & t_{0} \leqslant t \leqslant b\end{cases}
$$


(ii) If $\sigma_{L}>\sigma_{R}$ then

$$
\alpha(t, \varepsilon)=\left\{\begin{array}{cc}
u_{L}(t)+w(t, \varepsilon)+p_{1} p_{2}^{-1} w^{\prime}(a, \varepsilon), & a \leqslant t \leqslant t_{0}, \\
u_{R}(t)+w(t, \varepsilon)-q_{2} q_{1}^{-1} w^{\prime}(b, \varepsilon), & t_{0} \leqslant t \leqslant b, \\
\beta(t, \varepsilon)=u_{1}(t), \quad a \leqslant t \leqslant b . &
\end{array}\right.
$$

In case (i), for example, $\varepsilon u_{L}^{\prime \prime}=f\left(u_{L}^{\prime}\right), \varepsilon u_{R}^{\prime \prime}=f\left(u_{R}^{\prime}\right)$ in $(a, b)$ and $q_{1} u_{L}(b)+q_{2} \sigma_{L} \leqslant B$, $p_{1} u_{R}(a)-p_{2} \sigma_{R} \leqslant A$, and consequently $\alpha(t, \varepsilon)=u_{1}(t)=\max \left\{u_{L}(t), u_{R}(t)\right\}$ is a lower solution (cf. [11]). Moreover, with $w$ as before, $\beta^{\prime}\left(t_{0}^{-}, \varepsilon\right)=\beta^{\prime}\left(t_{0}^{+}, \varepsilon\right)=$ $\frac{1}{2}\left(\sigma_{L}+\sigma_{R}\right)$ and $\varepsilon \beta^{\prime \prime} \leqslant f\left(\beta^{\prime}\right)$ for $t$ in $\left(a, t_{0}\right) \cup\left(t_{0}, b\right)$, that is, $\beta$ is an upper solution. Finally it is easy to see that $y^{\prime}(t, \varepsilon)=\sigma_{L}+\vartheta\left(w^{\prime}(t, \varepsilon)\right)$ in $\left[a, t_{0}\right]$ and $y^{\prime}(t, \varepsilon)=\sigma_{R}+$ $\vartheta\left(w^{\prime}(t, \varepsilon)\right)$ in $\left[t_{0}, b\right]$. Thus the conclusion of the theorem follows from Heidel's theorem [7]. Case (ii) is handled similarly.

Before discussing some examples we make several remarks.

REMARK 3.1. If $u=u_{R}(t)$ is a solution of the reduced problem $\left(\Re_{R}\right)$ then a necessary condition that $u_{R}$ be stable in the sense described in Theorem 3.2 is that $f^{\prime}\left(\sigma_{R}\right) \leqslant 0$. Similarly a solution $u=u_{L}(t)$ of $\left(R_{L}\right)$ can be stable only if $f^{\prime}\left(\sigma_{L}\right) \geqslant 0$.

REMARK 3.2. The boundary layer function $w_{L}$ is estimated very easily if there is a positive constant $k$ such that $f^{\prime}\left(\sigma_{R}\right) \leqslant-k<0$. It is not difficult to see that in the case of Theorems 3.1 and 3.2 we can take $w_{L}(t, \varepsilon) \approx \varepsilon k_{1}^{-1}\left(\sigma_{R}+A\right) e^{-k_{1}(t-a) \varepsilon^{-1}}$ and $w_{L}(t, \varepsilon) \approx-\varepsilon k_{1}^{-1} p_{2}^{-1}\left(p_{1} u_{R}(a)-p_{2} \sigma_{R}-A\right) e^{-k_{1}(t-a) \varepsilon^{-1}}$, respectively, for a positive constant $k_{1} \leqslant k$.

Similarly, in the case of Theorem 3.3, if there is a positive constant $k$ such that $f^{\prime}\left(\sigma_{L}\right) \geqslant k>0$ and $f^{\prime}\left(\sigma_{R}\right) \leqslant-k<0$ then the interior layer function $w$ is estimated by

$$
w(t, \varepsilon) \approx u_{L}(t)+\frac{1}{2} \varepsilon k_{1}^{-1}\left(\sigma_{R}-\sigma_{L}\right) e^{k_{1}\left(t-t_{0}\right) \varepsilon^{-1}}
$$

for $t$ in $\left[a, t_{0}\right]$ and

$$
w(t, \varepsilon) \approx u_{R}(t)+\frac{1}{2} \varepsilon k_{1}^{-1}\left(\sigma_{R}-\sigma_{L}\right) e^{-k_{1}\left(t-t_{0}\right) \varepsilon^{-1}}
$$

for $t$ in $\left[t_{0}, b\right]$.

REMARK 3.3. Our basic assumption that $f\left(y^{\prime}\right)=\theta\left(\left|y^{\prime}\right|^{n}\right)$ as $\left|y^{\prime}\right| \rightarrow \infty$ for $n>2$ deserves a brief comment in view of the results of this section which imply that the solutions of $\left(\Re_{1}\right)$ have uniformly bounded first derivatives. It is meant to emphasize two aspects of this problem and the more general one considered in the following sections. First, for such right-hand sides $f$ the Dirichlet problem is essentially ill-posed (cf. [4], [17, Chapter II] and Example 3.1) and so the "correct" boundary conditions are the ones under study here. Second, as the results of $\$ 2$ show, the right-hand side must have sufficiently many zeros in order for the asymptotic theory to be applicable. To fix the ideas of this paper it may be helpful if the reader regards $f\left(t, y, y^{\prime}\right)$ as a polynomial in $y^{\prime}$ of degree at least three.

REMARK 3.4. The assumption regarding the positivity of $p_{1}$ and $q_{1}$ is necessary for the validity of Theorem 3.3 (cf. Example 3.3).

REMARK 3.5. There is a connection between the nonoccurrence of boundary layer behavior as described by Theorem 3.2 and the occurrence of interior layer 
behavior as described by Theorem 3.3. Suppose for simplicity that $p_{1}=q_{1}=p_{2}=$ $q_{2}=1$ in $\left(\mathscr{T}_{1}\right)$ and suppose that the reduced problems $\left(\mathscr{R}_{L}\right)$ and $\left(\Re_{R}\right)$ have stable solutions $u=u_{L}(t)=\sigma_{L} t+c$ and $u=u_{R}(t)=\sigma_{R} t+c^{\prime}$ with $\sigma_{L}<\sigma_{R}$. If $u_{L}(b)+$ $\sigma_{L}<B$ and $u_{R}(a)-\sigma_{R}<A$ but $f\left(B-u_{L}(b)\right)<0$ and $f\left(u_{R}(a)-A\right)<0$ then Theorem 3.2 and its "reflected" version involving $\left(\Re_{L}\right)$ are inapplicable because the required inequalities are violated by such $A$ and $B$. We claim that if $\mid u_{L}(\tau)-$ $u_{R}(\tau) \mid$ is not too large for $\tau=a$ and $\tau=b$ then in fact $u_{L}(a)>u_{R}(a)$ and $u_{L}(b)<u_{R}(b)$, that is, $u_{L}$ and $u_{R}$ intersect at a point in $(a, b)$. To see this, note first that for $\omega=u_{R}(a)-u_{L}(a), 0>f\left(u_{R}(a)-A\right)=f\left(\sigma_{L}+\omega\right)$ and therefore the stability of $\sigma_{L}$ implies that $\omega<0$ if $|\omega|$ is not too large. Similarly, for $\nu=u_{R}(b)-u_{L}(b)$, $0>f\left(B-u_{L}(b)\right)=f\left(\sigma_{R}+\nu\right)$, and so the stability of $\sigma_{R}$ implies that $\nu>0$ if $|\nu|$ is not too large. Thus there is a chance that Theorem 3.3 will apply to the functions $u_{L}$ and $u_{R}$ if $f(\lambda)>0$ for $\lambda$ in $\left(\sigma_{L}, \sigma_{R}\right)$. This inequality is certainly satisfied if $\sigma_{L}$ and $\sigma_{R}$ are adjacent stable zeros of $f$.

On the other hand, if $\sigma_{L}>\sigma_{R}, u_{L}(b)+\sigma_{L}>B, f\left(B-u_{L}(b)\right)>0, u_{R}(a)-\sigma_{R}>$ $A$ and $f\left(u_{R}(a)-A\right)>0$ then it follows as before that $u_{L}(a)<u_{R}(a)$ and $u_{L}(b)>$ $u_{R}(b)$. Consequently $u_{L}$ and $u_{R}$ intersect in $(a, b)$ and we are led again to consider the possibility of a crossing as described by Theorem 3.3.

We turn now to a discussion of several examples which illustrate the theory of this section.

EXAMPLE 3.1. Consider first the problem

$$
\begin{gathered}
\varepsilon y^{\prime \prime}=-y^{\prime}-y^{\prime 3}, \quad 0<t<1, \\
p y(0, \varepsilon)-y^{\prime}(0, \varepsilon)=A, \quad y(1, \varepsilon)=B,
\end{gathered}
$$

for $p \geqslant 0$. The reduced equation $f(\sigma)=-\sigma-\sigma^{3}=0$ has $\sigma=0$ as its only real solution and since $f^{\prime}(0)=-1$ we make the corresponding reduced solution $u$ satisfy $u(1)=B$ (cf. Remark 3.1$)$, that is, we consider $u=u_{R}(t) \equiv B$. Suppose first that $p=0$. If $A=0$ then $y(t, \varepsilon) \equiv B$ is the solution of (E1). However, if $A \neq 0$ then $A f(\lambda)=-A \lambda\left(1+\lambda^{2}\right)>0$ for $\lambda$ in $(0,-A]$. Consequently we deduce from Theorem 3.1 that for all $A$ the problem (E1) has a unique solution $y=y(t, \varepsilon)$ such that $y(t, \varepsilon)=B+\vartheta\left(\varepsilon|A| e^{-t \varepsilon^{-1}}\right)$ in [0,1]. Finally if $p>0$ then for $A=p B, y(t, \varepsilon)$ $\equiv B$ is the solution of (E1), while if $A \neq p B$ then

$$
(p B-A) f(\lambda)=-\lambda(p B-A)\left(1+\lambda^{2}\right)<0
$$

for $\lambda$ in $(0, p B-A]$. Thus by Theorem 3.2 the problem (E1) has a unique solution $y=y(t, \varepsilon)$ for all $A$ and $B$ such that $y(t, \varepsilon)=B+\vartheta\left(\varepsilon|p B-A| e^{-t \varepsilon^{-1}}\right)$ in $[0,1]$.

We note that the Dirichlet problem (cf. [1], [4]) $\varepsilon y^{\prime \prime}=-y^{\prime}-y^{\prime 3}, 0<t<1$, $y(0, \varepsilon)=A, y(1, \varepsilon)=B$, has no solution if $A \neq B$ and $\varepsilon>0$ is sufficiently small.

EXAMPLE 3.2. Consider next the problem

$$
\begin{gathered}
\varepsilon y^{\prime \prime}=y^{\prime}-y^{\prime 3}, \quad 0<t<1, \\
y(0, \varepsilon)-y^{\prime}(0, \varepsilon)=A, \quad y(1, \varepsilon)+y^{\prime}(1, \varepsilon)=B .
\end{gathered}
$$

The reduced equation $f\left(u^{\prime}\right)=u^{\prime}-u^{\prime 3}=0$ has now three solutions $u_{1}^{\prime}=1, u_{2}^{\prime}=$ -1 and $u_{3}^{\prime}=0$ which are such that $f^{\prime}( \pm 1)=-2$ and $f^{\prime}(0)=1$. Thus we make $u_{1}$ and $u_{2}$ satisfy $u_{j}(1)+u_{j}^{\prime}(1)=B$ for $j=1,2$, that is, $u_{1}(t)=t+B-2$ and 
$u_{2}(t)=-t+B+2$, and we make $u_{3}$ satisfy $u_{3}(0)-u_{3}^{\prime}(0)=A$, that is, $u_{3}(t) \equiv A$. Consider first $u_{1}$. If $A=B-3$ then $u_{1}(0)-u_{1}^{\prime}(0)=A$ and therefore $y(t, \varepsilon)=t+$ $B-2$ is the solution of (E2). However if $A<B-3$ then $\left(u_{1}(0)-1-A\right) f(\lambda)=$ $(B-3-A) \lambda\left(1-\lambda^{2}\right)<0$ for $\lambda$ in $(1, B-2-A]$ and so we apply Theorem 3.2 to deduce that the problem (E2) has a unique solution $y=y(t, \varepsilon)$ such that $y(t, \varepsilon)=u_{1}(t)+\mathcal{O}\left(\frac{1}{2} \varepsilon(B-3-A) e^{-2 t \varepsilon^{-1}}\right)$ in [0,1]. Finally if $A>B-3$ we have that $(B-3-A) \lambda\left(1-\lambda^{2}\right)<0$ for $\lambda$ in $[B-2-A, 1)$ provided that $B-2-A$ $>0$. Again from Theorem 3.2 we deduce the existence of a unique solution $y=y(t, \varepsilon)$ of (E2) (with $B-3<A<B-2$ ) such that in $[0,1], y(t, \varepsilon)=u_{1}(t)+$ $\vartheta\left(k_{1}^{-1} \varepsilon|B-3-A| e^{-k_{1} t \varepsilon^{-1}}\right)$ for a positive constant $k_{1}<2$.

The asymptotic behavior described by the function $u_{2}$ is clearly a reflection of that described by $u_{1}$. Therefore if $B+3 \leqslant A$ problem (E2) has a unique solution $y=y(t, \varepsilon)$ such that $y(t, \varepsilon)=u_{2}(t)+\vartheta\left(\frac{1}{2} \varepsilon|B+3-A| e^{-2 t \varepsilon^{-1}}\right)$ in $[0,1]$. While if $B+2<A<B+3$ the solution $y(t, \varepsilon)$ satisfies

$$
y(t, \varepsilon)=u_{2}(t)+\mathcal{O}\left(k_{1}^{-1} \varepsilon(B+3-A) e^{-k_{1} t \varepsilon^{-1}}\right)
$$

in $[0,1]$ for a positive constant $k_{1}<2$.

Next consider the function $u_{3} \equiv A$. If $A=B$ then $y(t, \varepsilon) \equiv A$ is the solution of (E2), while if $A<B,(A-B) f(\lambda)=(A-B) \lambda\left(1-\lambda^{2}\right)<0$ for $\lambda$ in $(0, B-A]$ if $B-A<1$, and if $A>B,(A-B) f(\lambda)<0$ for $\lambda$ in $[B-A, 0)$ if $B-A>-1$. Thus for $B-1<A<B+1$ we deduce from the reflected version of Theorem 3.2 the existence of a unique solution $y=y(t, \varepsilon)$ of (E2) such that in $[0,1], y(t, \varepsilon)=A$ $+\mathcal{O}\left(k_{1}^{-1} \varepsilon|B-A| e^{-k_{1}(1-t) \varepsilon^{-1}}\right)$ for a positive constant $k_{1}<1$.

Note that we have proved the existence of a solution of (E2) for all boundary values $A$ and $B$ except those satisfying the inequalities $B-2 \leqslant A \leqslant B-1$ and $B+1 \leqslant A \leqslant B+2$. These are precisely the boundary values for which the boundary layer behavior described by Theorem 3.2 is impossible. Thus (cf. Remark 3.5) we are led to consider the "angular" paths

$$
u_{4}(t)=\left\{\begin{array}{ll}
u_{3}(t), & 0 \leqslant t \leqslant t_{0}, \\
u_{1}(t), & t_{0} \leqslant t \leqslant 1,
\end{array} \text { and } \quad u_{5}(t)= \begin{cases}u_{3}(t), & 0 \leqslant t \leqslant \tilde{t}_{0} \\
u_{2}(t), & \tilde{t}_{0} \leqslant t \leqslant 1\end{cases}\right.
$$

It follows directly that $t_{0}=A-B+2$ belongs to $(0,1)$ if and only if $B-2<A$ $<B-1$ while $\tilde{t}_{0}=B-A+2$ belongs to $(0,1)$ if and only if $B+1<A<B+$ 2. Consider first $u_{4}$. For $\sigma_{L}=0$ and $\sigma_{R}=1$ we see that $\left(\sigma_{R}-\sigma_{L}\right) f(\lambda)=\lambda\left(1-\lambda^{2}\right)$ $>0$ for $\lambda$ in $(0,1)$ and so Theorem 3.3 allows us to deduce the existence of a solution $y=y(t, \varepsilon)$ of (E2) for $B-2<A<B-1$ such that in $[0,1], y(t, \varepsilon)=$ $u_{4}(t)+\theta\left(\frac{1}{2} k_{1}^{-1} \varepsilon e^{-k_{1}\left|t-t_{0}\right| \varepsilon^{-1}}\right)$ with $0<k_{1}<1$. Similarly, in the case of $u_{5}$, for $\sigma_{L}=0$ and $\sigma_{R}=-1$ we see that $\left(\sigma_{R}-\sigma_{L}\right) f(\lambda)=-\lambda\left(1-\lambda^{2}\right)>0$ for $\lambda$ in $(-1,0)$ and so the problem (E2) for $B+1<A<B+2$ has a solution $y=y(t, \varepsilon)$ such that in $[0,1], y(t, \varepsilon)=u_{5}(t)+\vartheta\left(\frac{1}{2} k_{1}^{-1} \varepsilon e^{-k_{1}\left|t-i_{0}\right| e^{-1}}\right)$.

Finally if $A=B-2$ then it is easy to show that (E2) has a solution $y=y(t, \varepsilon)$ such that $y(t, \varepsilon) \rightarrow t+B-2$ as $\varepsilon \rightarrow 0^{+}$(as expected). Similarly if $A=B-1$ or 
$A=B+1$ a solution $y$ exists and satisfies $y(t, \varepsilon) \rightarrow 0$ as $\varepsilon \rightarrow 0^{+}$, while if $A=B+$ 2 then $y(t, \varepsilon) \rightarrow-t+B+2$ as $\varepsilon \rightarrow 0^{+}$. The convergence is of course uniform in $[0,1]$ for these choices of $A$ and $B$.

EXAMPLE 3.3. In this final example we illustrate the remark that Theorem 3.3 is not necessarily valid if either $p_{1}=0$ or $q_{1}=0$. The problem is

$$
\varepsilon y^{\prime \prime}=1-y^{4}, \quad 0<t<1, \quad-y^{\prime}(0)=1, \quad y(1)=0,
$$

which has the unique solution $y(t, \varepsilon)=1-t$ for all $\varepsilon$. Consider however the "angular" path defined by $u_{1}(t)=u_{L}(t)=-t$ for $0 \leqslant t \leqslant \frac{1}{2}$ and $u_{1}(t)=u_{R}(t)=t$

-1 for $\frac{1}{2} \leqslant t \leqslant 1$. The functions $u_{L}$ and $u_{R}$ are stable in the sense that $f^{\prime}\left(u_{L}^{\prime}\right)=4$ $>0$ and $f^{\prime}\left(u_{R}^{\prime}\right)=-4<0$. Moreover, $\left(\sigma_{R}-\sigma_{L}\right) f(\lambda)=2\left(1-\lambda^{4}\right)>0$ for $|\lambda|<1$. Nevertheless there is no solution of (E3) which is close to $u_{1}(t)$ in $[0,1]$.

4. The general problem. In this section we discuss several results for the general problem

$$
\begin{gathered}
\varepsilon y^{\prime \prime}=f\left(t, y, y^{\prime}\right), \\
p_{1} y(a, \varepsilon)-p_{2} y^{\prime}(a, \varepsilon)=A, \quad q_{1} y(b, \varepsilon)+q_{2} y^{\prime}(b, \varepsilon)=B,
\end{gathered}
$$

for constants $p_{1}, p_{2}, q_{1}$ and $q_{2}$ with the same properties as in $\S 3$. The function $f$ is assumed to be at least continuous for all $t$ in $[a, b]$ and for all values of $y$ and $y^{\prime}$ under consideration. Moreover, for $(t, y)$ in compact subsets of $[a, b] \times \mathbf{R}$, $f\left(t, y, y^{\prime}\right)=\theta\left(\left|y^{\prime}\right|^{n}\right)$ as $\left|y^{\prime}\right| \rightarrow \infty$ for $n>2$. Recalling our results in $\S 3$ we now define certain reduced problems whose solutions we will use to study the existence and the asymptotic behavior of solutions of $(\mathscr{N})$, namely

$$
\begin{gathered}
f\left(t, u, u^{\prime}\right)=0, \quad a<t<t_{L} \leqslant b, \\
p_{1} u(a)-p_{2} u^{\prime}(a)=A, \\
f\left(t, u, u^{\prime}\right)=0, \quad a \leqslant t_{R}<t<b, \\
q_{1} u(b)+q_{2} u^{\prime}(b)=B,
\end{gathered}
$$

and

$$
f\left(t, u, u^{\prime}\right)=0, \quad a<t<b .
$$

Solutions of $\left(\Re_{L}\right),\left(\mathscr{R}_{R}\right)$ and $(\Re)$ will be denoted by $u_{L}, u_{R}$ and $u_{I}$ respectively.

Our experience with the simpler problem $\left(\Re_{1}\right)$ leads us to consider only solutions of these reduced problems which are stable in senses to be stated shortly. First we need to define some regions in $\left(t, y, y^{\prime}\right)$-space. In such regions we will seek solutions of the problem $(\mathcal{N})$ which are uniformly close to certain reduced paths (that is, curves consisting of solutions of $\left(\Re_{L}\right),\left(\Re_{R}\right)$ and/or $\left.(\Re)\right)$. However the derivative of a solution of $(\mathcal{K})$ may differ from the derivative of the corresponding reduced path at one or more points in $[a, b]$ by an amount of order one (cf. \$3), and therefore we must allow for this in the following definitions. Let a solution $u=u_{R}(t)$ exist in $[a, b]$ and let $\omega_{R}=p_{1} u_{R}(a)-p_{2} u_{R}^{\prime}(a)$. Then (cf. Theorem 3.2) we define the domain $\mathscr{D}\left(u_{R}\right)$ as follows. (Here and below $\delta$ denotes a generic small positive constant.)

$$
\mathscr{D}\left(u_{R}\right)=\left\{\left(t, y, y^{\prime}\right): a \leqslant t \leqslant b,\left|y-u_{R}(t)\right| \leqslant \delta,\left|y^{\prime}-u_{R}^{\prime}(t)\right| \leqslant d_{R}(t)\right\}
$$


where $d_{R}$ is a smooth positive function such that if $p_{2}>0$ then $p_{2}^{-1}\left|A-\omega_{R}\right| \leqslant$ $d_{R}(t) \leqslant p_{2}^{-1}\left|A-\omega_{R}\right|+\delta$ for $a \leqslant t \leqslant a+\delta / 2$ and $d_{R}(t) \leqslant \delta$ for $a+\delta \leqslant t \leqslant b$, while if $p_{2}=0$ then $d_{R}(t) \leqslant \delta$ in $[a, b]$.

We will also consider solution paths of the form (cf. Theorem 3.3)

$$
\begin{gathered}
u_{1}(t)= \begin{cases}u_{L}(t), & \left.a \leqslant t \leqslant t_{0}\left(<t_{L}\right), \quad \text { if } t_{L}>t_{R}\right) \\
u_{R}(t), & \left(t_{R}<\right) t_{0} \leqslant t \leqslant b,\end{cases} \\
u_{2}(t)= \begin{cases}u_{L}(t), & a \leqslant t \leqslant t_{1}, \\
u_{I}(t), & t_{1} \leqslant t \leqslant t_{2}, \\
u_{R}(t), & t_{2} \leqslant t \leqslant b,\end{cases}
\end{gathered}
$$

and

$$
u_{3}(t)= \begin{cases}u_{I}(t), & a \leqslant t \leqslant t_{2}, \\ u_{R}(t), & t_{2} \leqslant t \leqslant b .\end{cases}
$$

Here $u=u_{L}(t)$ is a solution of the reduced problem $\left(\Re_{L}\right)$ and $u=u_{I}(t)$ a solution of the reduced equation $(R)$ which we assume exist on the stated intervals. We define the following domains.

$$
\mathscr{D}\left(u_{1}\right)=\left\{\left(t, y, y^{\prime}\right): a \leqslant t \leqslant b,\left|y-u_{1}(t)\right| \leqslant \delta,\left|y^{\prime}-u_{1}^{\prime}(t)\right| \leqslant d_{1}(t)\right\}
$$

where $d_{1}$ is a smooth positive function such that $\left|u_{L}^{\prime}\left(t_{0}\right)-u_{R}^{\prime}\left(t_{0}\right)\right| \leqslant d_{1}(t) \leqslant \mid u_{L}^{\prime}\left(t_{0}\right)$ $-u_{R}^{\prime}\left(t_{0}\right) \mid+\delta$ for $t_{0}-\delta / 2 \leqslant t \leqslant t_{0}+\delta / 2$ and $d_{1}(t) \leqslant \delta$ for $t$ in $\left[a, t_{0}-\delta\right] \cup\left[t_{0}\right.$ $+\delta, b]$

$$
\mathscr{D}\left(u_{2}\right)=\left\{\left(t, y, y^{\prime}\right): a \leqslant t \leqslant b,\left|y-u_{2}(t)\right| \leqslant \delta,\left|y^{\prime}-u_{2}^{\prime}(t)\right| \leqslant d_{2}(t)\right\}
$$

where $d_{2}$ is a smooth positive function such that $\left|u_{L}^{\prime}\left(t_{1}\right)-u_{I}^{\prime}\left(t_{1}\right)\right| \leqslant d_{2}(t) \leqslant \mid u_{L}^{\prime}\left(t_{1}\right)$ $-u_{I}^{\prime}\left(t_{1}\right) \mid+\delta$ for $t_{1}-\delta / 2 \leqslant t \leqslant t_{1}+\delta / 2, \quad\left|u_{I}^{\prime}\left(t_{2}\right)-u_{R}^{\prime}\left(t_{2}\right)\right| \leqslant d_{2}(t) \leqslant \mid u_{I}^{\prime}\left(t_{2}\right)-$ $u_{R}^{\prime}\left(t_{2}\right) \mid+\delta$ for $t_{2}-\delta / 2 \leqslant t \leqslant t_{2}+\delta / 2$ and $d_{2}(t) \leqslant \delta$ for $t$ in $\left[a, t_{1}-\delta\right] \cup\left[t_{1}+\right.$ $\left.\delta, t_{2}-\delta\right] \cup\left[t_{2}+\delta, b\right]$ and

$$
\mathscr{D}\left(u_{3}\right)=\left\{\left(t, y, y^{\prime}\right): a \leqslant t \leqslant b,\left|y-u_{3}(t)\right| \leqslant \delta,\left|y^{\prime}-u_{3}^{\prime}(t)\right| \leqslant d_{3}(t)\right\}
$$

where $d_{3}$ is a smooth positive function such that $\left|u_{I}^{\prime}\left(t_{2}\right)-u_{R}^{\prime}\left(t_{2}\right)\right| \leqslant d_{3}(t) \leqslant \mid u_{I}^{\prime}\left(t_{2}\right)$ $-u_{R}^{\prime}\left(t_{2}\right) \mid+\delta$ for $t_{2}-\delta / 2 \leqslant t \leqslant t_{2}+\delta / 2, d_{3}(t) \leqslant \delta$ for $t$ in $\left[a+\delta, t_{2}-\delta\right] \cup\left[t_{2}\right.$ $+\delta, b]$, and $d_{3}(t)=d_{R}(t)$ for $t$ in $[a, a+\delta / 2]$ with $u_{R}$ replaced by $u_{1}$.

Finally if $u$ is any one of the solutions or solution paths defined above then we define the domain $\mathscr{D}_{\delta}(u)$ as

$$
\mathscr{D}_{\delta}(u)=\left\{\left(t, y, y^{\prime}\right): a \leqslant t \leqslant b,|y-u(t)| \leqslant \delta,\left|y^{\prime}-u^{\prime}(t)\right| \leqslant \delta\right\} .
$$

We now define the various types of stability which solutions of the reduced problems can possess (cf. [8] and Remarks 3.1 and 3.2 above for motivation). The essential idea behind these definitions is that if a reduced path either fails to satisfy the boundary condition at an endpoint or if it is not differentiable at a point in $(a, b)$, then the derivative of the solution of $(\mathscr{T})$ under study behaves nonuniformly there as $\varepsilon \rightarrow 0^{+}$. This much is already clear from $\$ 3$. Consequently, in a neighborhood of such a point we must supplement the reduced path with layer terms (such as $w_{L}$ or $w$ above). Sufficient conditions for doing this are embodied in the 
definitions of stability which follow. We apologize for their somewhat technical appearance; however, they are nothing more than straightforward extensions of the stability concepts introduced in $\$ \S 2$ and 3 .

In what follows the function $f$ is assumed to be continuously differentiable with respect to $y$ and $y^{\prime}$ in the appropriate domain. Also if $\mathscr{D}$ is any one of the domains defined above and $J$ is any subinterval of $[a, b]$ then the notation $\mathscr{D} \cap J$ is used as an abbreviation for $\mathscr{D} \cap\left(J \times \mathbf{R}^{2}\right)$.

Definition 4.1. A solution $u=u_{R}(t)$ of $\left(\Re_{R}\right)$ which exists in $[a, b]$ is said to be strongly (weakly) $y^{\prime}$-stable if there is a positive constant $k$ such that $f_{y^{\prime}} \leqslant-k<0$, $\left(f_{y^{\prime}} \leqslant 0\right)$ in $\mathscr{D}_{\delta}\left(u_{R}\right)$.

Definition 4.2. A solution $u=u_{I}(t)$ of $(\Re)$ which exists in $[a, b]$ is said to be locally strongly (weakly) $y^{\prime}$-stable if there is a positive constant $k$ such that $f_{y^{\prime}} \leqslant-k$ $<0\left(f_{y^{\prime}} \leqslant 0\right)$ in $\mathscr{D}_{\delta}\left(u_{1}\right) \cap[a, a+\delta]$ if $p_{1} u_{I}(a)-p_{2} u_{I}^{\prime}(a) \neq A$ with $p_{2}>0$ and $f_{y^{\prime}} \geqslant k>0\left(f_{y^{\prime}} \geqslant 0\right)$ in $\mathscr{D}_{\delta}\left(u_{I}\right) \cap[b-\delta, b]$ if $q_{1} u_{I}(b)+q_{2} u_{I}^{\prime}(b) \neq B$ with $q_{2}>0$.

Definition 4.3. A solution path $u=u_{1}(t)$ with $u_{L}^{\prime}\left(t_{0}\right) \neq u_{R}^{\prime}\left(t_{0}\right)$ is said to be strongly (weakly) $y^{\prime}$-stable if there is a positive constant $k$ such that $f_{y^{\prime}} \geqslant k>0\left(f_{y^{\prime}} \geqslant 0\right)$ in $\mathscr{D}_{\delta}\left(u_{1}\right) \cap\left[a, t_{0}\right]$ and $f_{y^{\prime}} \leqslant-k<0\left(f_{y^{\prime}} \leqslant 0\right)$ in $\mathscr{D}_{\delta}\left(u_{1}\right) \cap\left[t_{0}, b\right]$.

Definition 4.4. A solution path $u=u_{2}(t)$ with $u_{L}^{\prime}\left(t_{1}\right) \neq u_{I}^{\prime}\left(t_{1}\right)$ and $/$ or $u_{I}^{\prime}\left(t_{2}\right) \neq$ $u_{R}^{\prime}\left(t_{2}\right)$ is said to be strongly (weakly) $y^{\prime}$-stable if there is a positive constant $k$ such that $f_{y^{\prime}} \geqslant k>0\left(f_{y^{\prime}} \geqslant 0\right)$ in $\mathscr{D}_{\delta}\left(u_{2}\right) \cap\left[t_{1}-\delta, t_{1}\right]$ and $f_{y^{\prime}} \leqslant-k<0\left(f_{y^{\prime}} \leqslant 0\right)$ in $\mathscr{D}_{\delta}\left(u_{2}\right) \cap\left[t_{1}, t_{1}+\delta\right]$ and $/$ or $f_{y^{\prime}} \geqslant k>0\left(f_{y^{\prime}} \geqslant 0\right)$ in $\mathscr{D}_{\delta}\left(u_{2}\right) \cap\left[t_{2}-\delta, t_{2}\right]$ and $f_{y^{\prime}} \leqslant$ $-k<0\left(f_{y^{\prime}} \leqslant 0\right)$ in $\mathscr{D}_{\delta}\left(u_{2}\right) \cap\left[t_{2}, t_{2}+\delta\right]$.

Definition 4.5. A solution path $u=u_{3}(t)$ is said to be locally strongly (weakly) $y^{\prime}$-stable if there is a positive constant $k$ such that $f_{y^{\prime}} \leqslant-k<0 \quad\left(f_{y^{\prime}} \leqslant 0\right)$ in $\mathscr{D}_{\delta}\left(u_{3}\right) \cap[a, a+\delta]$. Moreover, if $u_{I}^{\prime}\left(t_{2}\right) \neq u_{R}^{\prime}\left(t_{2}\right)$ then we require also that $f_{y^{\prime}} \geqslant$ $k>0\left(f_{y^{\prime}} \geqslant 0\right)$ in $\mathscr{D}_{\delta}\left(u_{3}\right) \cap\left[t_{2}-\delta, t_{2}\right]$ and $f_{y^{\prime}} \leqslant-k<0\left(f_{y^{\prime}} \leqslant 0\right)$ in $\mathscr{D}_{\delta}\left(u_{3}\right) \cap$ $\left[t_{2}, t_{2}+\delta\right]$.

The final definition of stability we will need involves the partial derivative $f_{y}$ and for this reason will be termed $y$-stability in conformity with the previous definitions of $y^{\prime}$-stability which involve $f_{y^{\prime}}$. This is a type of stability we have not encountered before in our study of $(\mathscr{R})$. It is used to guarantee that solutions of $(\mathscr{T})$ and their first derivatives are uniformly bounded in $[a, b]$ (cf. [16]) and also to permit the construction of layer terms when the reduced path is only weakly stable. More general definitions of $y$-stability are of ten needed and the reader can consult [8] or [9] for such definitions, as well as for further motivation.

Definition 4.6. A solution or solution path $u=u(t)$ is said to be $y$-stable if there is a positive constant $m$ such that $f_{y} \geqslant m>0$ in $D_{\delta}(u)$ with $y^{\prime}=u^{\prime}(t)$.

Using these definitions of stability we can begin our study of the nonlinear problem $(\mathfrak{T})$. In the theorems below we assume without stating so each time that the function $f$ is continuous in $\left(t, y, y^{\prime}\right)$ and continuously differentiable in $y$ and $y^{\prime}$ for all values of $t, y, y^{\prime}$ in the domain $\mathscr{D}(u)$ where $u$ is the reduced solution under consideration. Moreover, we tacitly assume that a solution of a reduced problem $\left(\Re_{L}\right),\left(\Re_{R}\right)$ or $(\Re)$ is of class $C^{(2)}$ in its interval of existence. (With regard to the "angular" path $u_{1}$ and possibly $u_{2}$ and $u_{3}$ we assume that the functions $u_{L}, u_{R}$ and 
$u_{I}$ which comprise these paths are of class $C^{(2)}$ in their respective intervals of existence.)

Our first result is the analog of Theorem 3.2 of the previous section and therefore we assume that $p_{2}>0$.

THEOREM 4.1. Assume that the reduced problem $\left(\Re_{R}\right)$ has a solution $u=u_{R}(t)$ which exists in $[a, b]$ and which is strongly or weakly $y^{\prime}$-stable and $y$-stable. Assume also that either $p_{1} u_{R}(a)-p_{2} u_{R}^{\prime}(a)=A$ or (if $\left.p_{1} u_{R}(a)-p_{2} u_{R}^{\prime}(a) \neq A\right)\left(p_{1} u_{R}(a)-\right.$ $\left.p_{2} u_{R}^{\prime}(a)-A\right) f\left(a, u_{R}(a), \lambda\right)<0$ for all $\lambda$ in $\left(u_{R}^{\prime}(a), p_{2}^{-1}\left(p_{1} u_{R}(a)-A\right)\right]$. Then there exists an $\varepsilon_{0}>0$ such that the problem $(\mathcal{K})$ with $p_{2}>0$ has a solution $y=y(t, \varepsilon)$ whenever $0<\varepsilon \leqslant \varepsilon_{0}$. In addition, for $t$ in $[a, b]$ we have that

$$
y(t, \varepsilon)=u_{R}(t)+\vartheta\left(w_{L}(t, \varepsilon)\right)+\vartheta(\varepsilon)
$$

and

$$
y^{\prime}(t, \varepsilon)=u_{R}^{\prime}(t)+\vartheta\left(w_{L}^{\prime}(t, \varepsilon)\right)+\vartheta(\varepsilon)
$$

where $w_{L}$ satisfies $w_{L}^{\prime}(a, \varepsilon)=p_{2}^{-1}\left(p_{1} u_{R}(a)-p_{2} u_{R}^{\prime}(a)-A\right), \lim _{\varepsilon \rightarrow 0^{+}} w_{L}(t, \varepsilon)=0$ for $a \leqslant t \leqslant b$ and $\lim _{\varepsilon \rightarrow 0^{+}} w_{L}^{\prime}(t, \varepsilon)=0$ for $a<t \leqslant b$.

Proof. Despite the general nature of the function $f$ the proof of this theorem is essentially a repetition of the proof of Theorem 3.1. Suppose for definiteness that $p_{1} u_{R}(a)-p_{2} u_{R}^{\prime}(a) \leqslant A$ and define for $a \leqslant t \leqslant b$ and $0<\varepsilon \leqslant \varepsilon_{0}$,

$$
\alpha(t, \varepsilon)=u_{R}(t)-\varepsilon \gamma m^{-1}, \quad \beta(t, \varepsilon)=u_{R}(t)+w_{L}(t, \varepsilon)+\varepsilon \gamma m^{-1},
$$

where $\gamma>0$ is a constant to be determined momentarily and the function $w_{L}>0$ has the above properties for $0<\varepsilon \leqslant \varepsilon_{0}$. Clearly $p_{1} \alpha(a, \varepsilon)-p_{2} \alpha^{\prime}(a, \varepsilon)<A \leqslant$ $p_{1} \beta(a, \varepsilon)-p_{2} \beta^{\prime}(a, \varepsilon)$ and $q_{1} \alpha(b, \varepsilon)+q_{2} \alpha^{\prime}(b, \varepsilon) \leqslant B \leqslant q_{1} \beta(b, \varepsilon)+q_{2} \beta^{\prime}(b, \varepsilon)$ by our choice of $w_{L}$. It is just as easy to see that $\varepsilon \alpha^{\prime \prime} \geqslant f\left(t, \alpha, \alpha^{\prime}\right)$ and $\varepsilon \beta^{\prime \prime} \leqslant f\left(t, \beta, \beta^{\prime}\right)$ in $(a, b)$ if $\gamma$ is chosen properly. Since $f\left(t, \sigma, \sigma^{\prime}\right)=f\left(t, u_{R}, u_{R}^{\prime}\right)+\left\{f\left(t, \sigma, u_{R}^{\prime}\right)-\right.$ $\left.f\left(t, u_{R}, u_{R}^{\prime}\right)\right\}+\left\{f\left(t, \sigma, \sigma^{\prime}\right)-f\left(t, \sigma, u_{R}^{\prime}\right)\right\}$ we have first that

$$
\begin{aligned}
\varepsilon \alpha^{\prime \prime}-f\left(t, \alpha, \alpha^{\prime}\right) & =\varepsilon u_{R}^{\prime \prime}-f\left(t, u_{R}, u_{R}^{\prime}\right)+f_{y}\left(t, \xi_{1}, u_{R}^{\prime}\right) \varepsilon \gamma m^{-1} \\
& \geqslant-\varepsilon M+\varepsilon \gamma \geqslant 0 \text { if } \gamma \geqslant M=\max \left|u_{R}^{\prime \prime}\right| .
\end{aligned}
$$

(Here $\xi_{1}=u_{R}+\vartheta(\varepsilon)$ is the appropriate intermediate point.) Secondly,

$$
\begin{aligned}
f\left(t, \beta, \beta^{\prime}\right)-\varepsilon \beta^{\prime \prime}= & f\left(t, u_{R}, u_{R}^{\prime}\right)+f_{y}\left(t, \xi_{2}, u_{R}^{\prime}\right)\left[w_{L}+\varepsilon \gamma m^{-1}\right] \\
& +\left\{f\left(t, \beta, \beta^{\prime}\right)-f\left(t, \beta, u_{R}^{\prime}\right)\right\}-\varepsilon u_{R}^{\prime \prime}-\varepsilon w_{L}^{\prime \prime} .
\end{aligned}
$$

By the stability assumptions of the theorem the quantity $\{\cdot\}-\varepsilon w_{L}^{\prime \prime}$ is nonnegative in $[a, a+\delta)$ and of order $o(\nu(t, \varepsilon))$ in $[a+\delta, b]$ for $\nu(t, \varepsilon)=\max \left\{\varepsilon, w_{L}(t, \varepsilon)\right\}$ with $t$ in $[a+\delta, b]$. Therefore $f\left(t, \beta, \beta^{\prime}\right)-\varepsilon \beta^{\prime \prime} \geqslant 0$ in $(a, b)$ for $\gamma \geqslant M$.

The final step in the proof consists in establishing a bound on $y^{\prime}(t, \varepsilon)$ for a solution of $\varepsilon y^{\prime \prime}=f\left(t, y, y^{\prime}\right)$ satisfying $\alpha \leqslant y \leqslant \beta$. However it follows directly that $y^{\prime}(t, \varepsilon)=u_{R}^{\prime}(t)+\mathcal{O}\left(w_{L}^{\prime}(t, \varepsilon)\right)+\mathcal{O}(\varepsilon)$ by arguing as in the proof of Theorem 3.1 and using the $y^{\prime}$ - and $y$-stability of $u_{R}$. For example, if $\alpha_{1}(t, \varepsilon)=u_{R}(t)-l \varepsilon-$ $\mu\left(t_{0}-t\right)$ with $l>0$ and $\mu>0$ then

$$
f\left(t, \alpha_{1}, \alpha_{1}^{\prime}\right)=f\left(t, u_{R}, u_{R}^{\prime}\right)-f_{y}\left(t, \xi_{1}, u_{R}^{\prime}\right)\left[l \varepsilon+\mu\left(t_{0}-t\right)\right]+f_{y^{\prime}}\left(t, \alpha_{1}, \xi_{2}\right) \mu<0
$$


since $f_{y}>0$ and $f_{y^{\prime}} \leqslant 0$ for $\xi_{1}=u_{R}+\vartheta(\varepsilon)$ and $\xi_{2}=u_{R}^{\prime}+\vartheta(\mu)$.

Thus Theorem 4.1 follows from Heidel's theorem [7].

The result corresponding to Theorem 4.1 for the reduced solution $u=u_{L}(t)$ (with $q_{2}>0$ ) follows now by making the change of variable $t \rightarrow a+b-t$ and applying Theorem 4.1 to the transformed problem. We leave its precise formulation to the reader.

It is of ten the case with the nonlinear problems under consideration here that the reduced equation has solutions $u=u_{I}(t)$ which cannot be made to satisfy either boundary condition. However if $u_{I}$ is locally $y^{\prime}$-stable and $y$-stable then it is not unreasonable to expect that the problem $(\mathcal{K})$ with $p_{2}>0$ and $q_{2}>0$ has a solution $y=y(t, \varepsilon)$ which is approximated by $u_{I}$ in $[a, b]$. This follows because the nonuniform behavior of $y^{\prime}$ is confined to small neighborhoods of $t=a$ and/or $t=b$ where we have $y^{\prime}$-stability and because the $y$-stability of $u_{I}$ is global. These heuristic ideas are made precise in the next theorem which can be viewed as a combination of Theorem 4.1 and its reflected counterpart for $u_{L}$. The proof is omitted.

THEOREM 4.2. Assume that the reduced equation $(\Re)$ has a solution $u=u_{I}(t)$ which is locally strongly or weakly $y^{\prime}$-stable and $y$-stable. Assume also that $p_{1} u_{I}(a)-$ $p_{2} u_{I}^{\prime}(a)=A$ or $\left(p_{1} u_{I}(a)-p_{2} u_{I}^{\prime}(a)-A\right) f\left(a, u_{I}(a), \lambda\right)<0$ for all $\lambda$ in $\left(u_{I}^{\prime}(a), p_{2}^{-1}\left(p_{1} u_{I}(a)-A\right)\right]$ and that $q_{1} u_{I}(b)+q_{2} u_{I}^{\prime}(b)=B$ or $\left(q_{1} u_{I}(b)+q_{2} u_{I}^{\prime}(b)-\right.$ $B) f\left(b, u_{I}(b), \lambda\right)<0$ for all $\lambda$ in $\left(u_{I}^{\prime}(b), q_{2}^{-1}\left(B-q_{1} u_{I}(b)\right)\right]$. Then there exists an $\varepsilon_{0}>0$ such that the problem $(\mathcal{T})$ with $p_{2}>0$ and $q_{2}>0$ has a solution $y=y(t, \varepsilon)$ whenever $0<\varepsilon \leqslant \varepsilon_{0}$. In addition, for $t$ in $[a, b]$ we have that

$$
y(t, \varepsilon)=u_{I}(t)+\mathcal{O}\left(w_{L}(t, \varepsilon)\right)+\mathcal{O}\left(w_{R}(t, \varepsilon)\right)+\vartheta(\varepsilon)
$$

and

$$
y^{\prime}(t, \varepsilon)=u_{I}^{\prime}(t)+\mathcal{O}\left(w_{L}^{\prime}(t, \varepsilon)\right)+\mathcal{O}\left(w_{R}^{\prime}(t, \varepsilon)\right)+\mathcal{O}(\varepsilon),
$$

where $w_{L}$ has the properties given in the conclusion of Theorem 4.1 with $u_{R}$ replaced by $u_{I}$, and $w_{L}$ satisfies $w_{L}^{\prime}(b, \varepsilon)=q_{2}^{-1}\left(B-q_{1} u_{I}(b)-q_{2} u_{I}^{\prime}(b)\right), \lim _{\varepsilon \rightarrow 0^{+}} w_{L}(t, \varepsilon)=0$ for $a \leqslant t \leqslant b$ and $\lim _{\varepsilon \rightarrow 0^{+}} w_{L}^{\prime}(t, \varepsilon)=0$ for $a \leqslant t<b$.

We consider next the situation in which the reduced problems $\left(\mathscr{R}_{L}\right)$ and $\left(\mathscr{R}_{R}\right)$ have solutions $u_{L}$ and $u_{R}$ which intersect at a point $t_{0}$ in $(a, b)$. Later (cf. Remark 4.4) we will see that such behavior is related to the nonoccurrence of the type of boundary layer behavior described in Theorem 4.1. Recalling Theorem 3.3 we are led to the following theorem.

THEOREM 4.3. Assume that the reduced problems $\left(\Re_{L}\right)$ and $\left(\Re_{R}\right)$ have solutions $u=u_{L}(t)$ and $u=u_{R}(t)$ in $\left[a, t_{L}\right)$ and $\left(t_{R}, b\right)$ respectively with $t_{L}>t_{R}$ such that $u_{L}\left(t_{0}\right)=u_{R}\left(t_{0}\right)=c$ and $\sigma_{L}=u_{L}^{\prime}\left(t_{0}\right) \neq u_{R}^{\prime}\left(t_{0}\right)=\sigma_{R}$ at a point $t_{0}$ in $\left(t_{R}, t_{L}\right)$. Assume also that the path $u=u_{1}(t)$ is strongly or weakly $y^{\prime}$-stable and $y$-stable and that $\left(\sigma_{R}-\sigma_{L}\right) f\left(t_{0}, c, \lambda\right)>0$ for all $\lambda$ in $\left(\sigma_{L}, \sigma_{R}\right)$. Then there exists an $\varepsilon_{0}>0$ such that the problem $(\mathcal{T})$ has a solution $y=y(t, \varepsilon)$ whenever $0<\varepsilon \leqslant \varepsilon_{0}$. In addition, we have 
that

$$
\begin{aligned}
& y(t, \varepsilon)=u_{1}(t)+\vartheta(w(t, \varepsilon))+\vartheta(\varepsilon) \text { for } a \leqslant t \leqslant b, \\
& y^{\prime}(t, \varepsilon)=u_{L}^{\prime}(t)+\vartheta\left(w^{\prime}(t, \varepsilon)\right)+\vartheta(\varepsilon) \text { for } a \leqslant t \leqslant t_{0}
\end{aligned}
$$

and

$$
y^{\prime}(t, \varepsilon)=u_{R}^{\prime}(t)+\mathcal{O}\left(w^{\prime}(t, \varepsilon)\right)+\mathcal{O}(\varepsilon) \text { for } t_{0} \leqslant t \leqslant b
$$

where the continuous function $w$ satisfies $w^{\prime}\left(t_{0}^{-}, \varepsilon\right)=\frac{1}{2}\left(\sigma_{R}-\sigma_{L}\right), w^{\prime}\left(t_{0}^{+}, \varepsilon\right)=$ $\frac{1}{2}\left(\sigma_{L}-\sigma_{R}\right), \lim _{\varepsilon \rightarrow 0^{+}} w(t, \varepsilon)=0$ for $a \leqslant t \leqslant b$ and $\lim _{\varepsilon \rightarrow 0^{+}} w^{\prime}(t, \varepsilon)=0$ for $a \leqslant t<$ $t_{0}$ and $t_{0}<t \leqslant b$.

Proof. Define for $a \leqslant t \leqslant b$ and $0<\varepsilon \leqslant \varepsilon_{0}$,

$$
\left.\begin{array}{l}
\alpha(t, \varepsilon)=u_{1}(t)-\varepsilon \gamma m^{-1} \\
\beta(t, \varepsilon)=u_{1}(t)+w(t, \varepsilon)+\varepsilon \gamma m^{-1}
\end{array}\right\} \quad \text { if } \sigma_{L}<\sigma_{R}
$$

and

$$
\left.\begin{array}{l}
\alpha(t, \varepsilon)=u_{1}(t)+w(t, \varepsilon)-\varepsilon \gamma m^{-1} \\
\beta(t, \varepsilon)=u_{1}(t)+\varepsilon \gamma m^{-1}
\end{array}\right\} \quad \text { if } \sigma_{L}>\sigma_{R}
$$

where $w$ has the above properties for $0<\varepsilon \leqslant \varepsilon_{0}$. Then one verifies easily that each of the inequalities of Heidel's theorem is valid. To obtain a bound on $y^{\prime}(t, \varepsilon)$ we estimate $y^{\prime}\left(t^{-}, \varepsilon\right)$ in $\left[t_{0}, b\right]$ as in Theorem 4.1 and $y^{\prime}\left(t^{+}, \varepsilon\right)$ in $\left[a, t_{0}\right]$ using the $y^{\prime}$ and $y$-stability of $u_{R}$ and $u_{L}$ respectively.

Suppose now that the reduced problems $\left(\Re_{L}\right),(\Re)$ and $\left(\Re_{R}\right)$ have solutions $u=u_{L}(t), u=u_{I}(t)$ and $u=u_{R}(t)$ such that $u_{L}\left(t_{1}\right)=u_{I}\left(t_{1}\right)$ and $u_{I}\left(t_{2}\right)=u_{R}\left(t_{2}\right)$ at distinct points $t_{1}$ and $t_{2}$ in $(a, b)$ with $t_{1}<t_{L}$ and $t_{R}<t_{2}$. If $u_{L}^{\prime}\left(t_{1}\right)=u_{I}^{\prime}\left(t_{1}\right)$ and $u_{I}^{\prime}\left(t_{2}\right)=u_{R}^{\prime}\left(t_{2}\right)$ it is clear (cf. the proof of Theorem 4.3) that if the path $u=u_{2}(t)$ is $y$-stable then the problem $(\mathcal{N})$ has a solution $y=y(t, \varepsilon)$ for $0<\varepsilon \leqslant \varepsilon_{0}$ such that $y(t, \varepsilon)=u_{2}(t)+\vartheta(\varepsilon)$ and $y^{\prime}(t, \varepsilon)=u_{2}^{\prime}(t)+\vartheta(\varepsilon)$ for $a \leqslant t \leqslant b$. However if $u_{L}^{\prime}\left(t_{1}\right)$ $\neq u_{I}^{\prime}\left(t_{1}\right)$ and $/$ or $u_{I}^{\prime}\left(t_{2}\right) \neq u_{R}^{\prime}\left(t_{2}\right)$ then we have the situation described in Theorem 4.3 at $t=t_{1}$ and $/$ or $t=t_{2}$. The proof of the following result can be patterned after the proof of Theorem 4.3.

THEOREM 4.4. Assume that the reduced problems $\left(\Re_{L}\right)$, $(\Re)$ and $\left(\Re_{R}\right)$ have solutions $u=u_{L}(t), u=u_{I}(t)$ and $u=u_{R}(t)$ such that $u_{L}\left(t_{1}\right)=u_{I}\left(t_{1}\right)=c_{1}, u_{I}\left(t_{2}\right)=$ $u_{R}\left(t_{2}\right)=c_{2}, \quad \sigma_{1}=u_{L}^{\prime}\left(t_{1}\right) \neq u_{I}^{\prime}\left(t_{1}\right)=\mu_{1}$ and $/$ or $\sigma_{2}=u_{I}^{\prime}\left(t_{2}\right) \neq u_{R}^{\prime}\left(t_{2}\right)=\mu_{2}$. Assume also that the path $u=u_{2}(t)$ is strongly or weakly $y^{\prime}$-stable and $y$-stable, and that $\left(\mu_{1}-\sigma_{1}\right) f\left(t_{1}, c_{1}, \lambda\right)>0$ for all $\lambda$ in $\left(\sigma_{1}, \mu_{1}\right)\left(\right.$ if $\left.\sigma_{1} \neq \mu_{1}\right)$ and $/$ or $\left(\mu_{2}-\sigma_{2}\right) f\left(t_{2}, c_{2}, \lambda\right)$ $>0$ for all $\lambda$ in $\left(\sigma_{2}, \mu_{2}\right)\left(\right.$ if $\left.\sigma_{2} \neq \mu_{2}\right)$. Then there exists an $\varepsilon_{0}>0$ such that the problem $(\Re)$ has a solution $y=y(t, \varepsilon)$ whenever $0<\varepsilon \leqslant \varepsilon_{0}$. In addition, we have that

$$
\begin{gathered}
y(t, \varepsilon)=u_{2}(t)+\vartheta\left(w_{1}(t, \varepsilon)\right)+\vartheta\left(w_{2}(t, \varepsilon)\right)+\vartheta(\varepsilon) \text { for } a \leqslant t \leqslant b, \\
y^{\prime}(t, \varepsilon)=u_{L}^{\prime}(t)+\vartheta\left(w_{1}^{\prime}(t, \varepsilon)\right)+\vartheta(\varepsilon) \text { for } a \leqslant t \leqslant t_{1}, \\
y^{\prime}(t, \varepsilon)=u_{1}^{\prime}(t)+\vartheta\left(w_{1}^{\prime}(t, \varepsilon)\right)+\vartheta\left(w_{2}^{\prime}(t, \varepsilon)\right)+\vartheta(\varepsilon) \text { for } t_{1} \leqslant t \leqslant t_{2}
\end{gathered}
$$


and

$$
y^{\prime}(t, \varepsilon)=u_{R}^{\prime}(t)+\Theta\left(w_{2}^{\prime}(t, \varepsilon)\right)+\Theta(\varepsilon) \text { for } t_{2} \leqslant t \leqslant b .
$$

Here $w_{j}(j=1,2)$ are continuous functions satisfying $w^{\prime}\left(t_{j}^{-}, \varepsilon\right)=\frac{1}{2}\left(\mu_{j}-\sigma_{j}\right)$, $w_{j}^{\prime}\left(t_{j}^{+}, \varepsilon\right)=\frac{1}{2}\left(\sigma_{j}-\mu_{j}\right), \lim _{\varepsilon \rightarrow 0^{+}} w_{j}(t, \varepsilon)=0$ for $a \leqslant t \leqslant b$ and $\lim _{\varepsilon \rightarrow 0^{+}} w_{j}^{\prime}(t, \varepsilon)=0$ for $a \leqslant t<t_{j}$ and $t_{j}<t \leqslant b$.

We consider finally the case in which the reduced equation $(\Re)$ has a solution $u=u_{I}(t)$ which intersects a solution $u_{R}$ of $\left(\Re_{R}\right)$ at a point in $\left(t_{R}, b\right)$. A similar result can be formulated if $u_{I}$ intersects a solution $u=u_{L}(t)$ of $\left(\Re_{L}\right)$ in $\left(a, t_{L}\right)$. We leave this as well as the proof of the following result to the reader.

THEOREM 4.5. Assume that the reduced problems $(\Re)$ and $\left(\Re_{R}\right)$ have solutions $u=u_{I}(t)$ and $u=u_{R}(t)$ such that $u_{I}\left(t_{2}\right)=u_{R}\left(t_{2}\right)=c_{2}$ at a point $t_{2}$ in $\left(t_{R}, b\right)$. Assume also that the path $u=u_{3}(t)$ is locally strongly or weakly $y^{\prime}$-stable and $y$-stable and that either $p_{1} u_{I}(a)-p_{2} u_{I}^{\prime}(a)=A$ or $\left(\right.$ if $\left.p_{1} u_{I}(a)-p_{2} u_{I}^{\prime}(a) \neq A\right)\left(p_{1} u_{I}(a)-p_{2} u_{I}^{\prime}(a)\right.$ $-A) f\left(a, u_{I}(a), \lambda\right)<0$ for all $\lambda$ in $\left(u_{I}^{\prime}(a), p_{2}^{-1}\left(p_{1} u_{I}(a)-A\right)\right]$. Assume finally that if $\sigma_{2}=u_{I}^{\prime}\left(t_{2}\right) \neq u_{R}^{\prime}\left(t_{2}\right)=\mu_{2}$ then $\left(\mu_{2}-\sigma_{2}\right) f\left(t_{2}, c_{2}, \lambda\right)>0$ for $\lambda$ in $\left(\sigma_{2}, \mu_{2}\right)$. Then there exists an $\varepsilon_{0}>0$ such that the problem $(\mathcal{U})$ with $p_{2}>0$ has a solution $y=y(t, \varepsilon)$ whenever $0<\varepsilon \leqslant \varepsilon_{0}$. In addition, we have that

$$
\begin{aligned}
& y(t, \varepsilon)=u_{3}(t)+\vartheta\left(w_{L}(t, \varepsilon)\right)+\vartheta\left(w_{2}(t, \varepsilon)\right)+\vartheta(\varepsilon) \text { for } a \leqslant t \leqslant b, \\
& y^{\prime}(t, \varepsilon)=u_{I}^{\prime}(t)+\vartheta\left(w_{L}^{\prime}(t, \varepsilon)\right)+\vartheta\left(w_{2}^{\prime}(t, \varepsilon)\right)+\vartheta(\varepsilon) \text { for } a \leqslant t \leqslant t_{2}
\end{aligned}
$$

and

$$
y^{\prime}(t, \varepsilon)=u_{R}^{\prime}(t)+\vartheta\left(w_{2}^{\prime}(t, \varepsilon)\right)+\vartheta(\varepsilon) \text { for } t_{2} \leqslant t \leqslant b .
$$

Here $w_{2}\left(w_{L}\right)$ has the properties given in the conclusion of Theorem 4.4 (Theorem 4.1 with $u_{R}$ replaced by $\left.u_{l}\right)$.

We close this section with several remarks.

REMARK 4.1. The boundary layer functions $w_{L}$ and $w_{R}$ are estimated very easily if $u_{R}$ and $u_{L}$ (or $u_{l}$ ) respectively are strongly (or locally strongly) $y^{\prime}$-stable. Namely we can take $w_{L}(t, \varepsilon) \approx-k_{1}^{-1} p_{2}^{-1} \varepsilon\left(p_{1} u(a)-p_{2} u^{\prime}(a)-A\right) e^{-k_{1}(t-a) \varepsilon^{-1}}$ for $u=u_{R}$ or $u_{I}$ and $w_{R}(t, \varepsilon) \approx k_{1}^{-1} q_{2}^{-1} \varepsilon\left(q_{1} u(b)+q_{2} u^{\prime}(b)-B\right) e^{-k_{1}(b-t) e^{-1}}$ for $u=u_{L}$ or $u_{l}$, where $k_{1}$ is a positive constant, $k_{1} \leqslant k$.

Similarly, the interior layer functions $w, w_{1}$ and $w_{2}$ are of exponential type if the reduced paths are strongly $y^{\prime}$-stable. For example, in the case of Theorem 4.3 we can approximate $w$ by $w(t, \varepsilon) \approx \frac{1}{2} k_{1}^{-1} \varepsilon\left(\sigma_{R}-\sigma_{L}\right) e^{k_{1}\left(t-t_{0}\right) e^{-1}}$ for $a \leqslant t \leqslant t_{0}$ and $w(t, \varepsilon) \approx \frac{1}{2} k_{1}^{-1} \varepsilon\left(\sigma_{R}-\sigma_{L}\right) e^{-k_{1}\left(t-t_{0}\right) \varepsilon^{-1}}$ for $t_{0} \leqslant t \leqslant b$, where $0<k_{1} \leqslant k$ (cf. [17], [13]).

REMARK 4.2. The $y$-stability of the various reduced solutions $u$ implies that the solutions of $(\mathcal{T})$ described above are locally unique in the sense that for each choice of $u$ there is only one solution $y$ of $(\mathcal{X})$ satisfying $\lim _{\varepsilon \rightarrow 0^{+}} y(t, \varepsilon)=u(t)$ in $[a, b]$ (cf. [16, Chapter 1]). However, for a given pair of boundary values $A$ and $B$ the problem $(\mathcal{C})$ may have more than one solution for all values of $\varepsilon>0$ sufficiently small (see Example 6.3). 
ReMARK 4.3. We note that it was not necessary to assume that $p_{1}>0$ and $q_{1}>0$ in the statement of Theorem 4.3 whereas these restrictions were required for the validity of Theorem 3.3. This is due to the fact that the path $u_{1}$ is assumed to be $y$-stable in $[a, b]$.

REMARK 4.4. There is also a connection between the occurrence of interior layer behavior and the nonoccurrence of boundary layer behavior for solutions of the general problem $(\mathcal{T}$ ) (cf. Remark 3.5). Suppose for example that the reduced problems $\left(\Re_{L}\right)$ and $\left(\Re_{R}\right)$ have strongly or weakly $y^{\prime}$-stable and $y$-stable solutions $u_{L}$ and $u_{R}$ such that

$$
\begin{gathered}
u_{L}(b)+u_{L}^{\prime}(b)<B \text { and } u_{R}(a)-u_{R}^{\prime}(a)<A \\
\text { but } \\
f\left(b, u_{L}(b), B-u_{L}(b)\right)<0 \text { and } f\left(a, u_{R}(a), u_{R}(a)-A\right)<0 .
\end{gathered}
$$

(Here we assume for simplicity that $p_{1}=q_{1}=p_{2}=q_{2}=1$ in $(\mathcal{T})$ ).) Then Theorem 4.1 and its reflected version are inapplicable. However, suppose that $\mid u_{L}(\tau)-$ $u_{R}(\tau) \mid$ is not too large for $\tau=a$ or $\tau=b$. We claim that

(i) $u_{L}(b)<u_{R}(b)$ and

(ii) $u_{L}(a)>u_{R}(a)$

that is, $u_{L}$ and $u_{R}$ intersect at least once in $(a, b)$. To verify inequality (i) set $\omega=u_{L}(b)-u_{R}(b)$ and note that

$$
\begin{aligned}
0 & >f\left(b, u_{L}(b), B-u_{L}(b)\right)=f\left(b, u_{R}(b)+\omega, u_{R}^{\prime}(b)-\omega\right) \\
& =f_{y}\left(b, u_{R}(b)+\theta \omega, u_{R}^{\prime}(b)-\omega\right) \omega+f\left(b, u_{R}(b), u_{R}^{\prime}(b)-\omega\right) .
\end{aligned}
$$

Suppose on the contrary that $u_{L}(b)>u_{R}(b)$, that is, $\omega>0$. Then the $y^{\prime}$-stability of $u_{R}$ implies that $f\left(b, u_{R}(b), u_{R}^{\prime}(b)-\omega\right) \geqslant 0$ while the positivity of $f_{y}$ implies that $f_{y} \omega>0$. Thus $f_{y} \omega+f\left(b, u_{R}(b), u_{R}^{\prime}(b)-\omega\right)>0$ which contradicts (4.2). Similarly, to verify inequality (ii) set $\nu=u_{R}(a)-u_{L}(a)$. Then we have that

$$
\begin{aligned}
0 & >f\left(a, u_{R}(a), u_{R}(a)-A\right)=f\left(a, u_{L}(a)+\nu, u_{L}^{\prime}(a)+\nu\right) \\
& =f_{y}\left(a, u_{L}(a)+\theta \nu, u_{L}^{\prime}(a)+\nu\right) \nu+f\left(a, u_{L}(a), u_{L}^{\prime}(a)+\nu\right) .
\end{aligned}
$$

Suppose on the contrary that $u_{L}(a)<u_{R}(a)$, that is, $\nu>0$. Then

$$
f\left(a, u_{L}(a), u_{L}^{\prime}(a)+\nu\right) \geqslant 0
$$

by the $y^{\prime}$-stability of $u_{L}$ while $f_{y} \nu>0$ by the positivity of $f_{y}$. Consequently $f_{y} \nu+f\left(a, u_{L}(a), u_{L}^{\prime}(a)+\nu\right)>0$, which contradicts (4.3).

Thus when the inequalities (4.1) obtain the functions $u_{L}$ and $u_{R}$ intersect in $(a, b)$ and we can check further to see if Theorem 4.3 is applicable. Such will always be the case if $u_{L}^{\prime}\left(t_{0}\right)$ and $u_{R}^{\prime}\left(t_{0}\right)\left(u_{L}\left(t_{0}\right)=u_{R}\left(t_{0}\right)=c\right)$ are adjacent zeros of $f\left(t_{0}, c, \sigma\right)$. Likewise if $u_{L}(b)+u_{L}^{\prime}(b)>B$ and $u_{R}(a)-u_{R}^{\prime}(a)>A$ but $f\left(b, u_{L}(b), B-u_{L}(b)\right)$ $>0$ and $f\left(a, u_{R}(a), u_{R}(a)-A\right)>0$ then $u_{L}$ and $u_{R}$ intersect at least once in $(a, b)$ and there is the possibility of a crossing as described by Theorem 4.3.

5. Some singular phenomena. The results of the previous section are distinguished by the fact that the convergence of a solution of the problem $(\Re)$ to a reduced solution takes place under the assumption of strong stability either at a boundary point or at an interior point. Namely, in the case of boundary layer behavior we 
required for example at $t=a$ that if $p_{1} u(a)-p_{2} u^{\prime}(a) \neq A$ then $\left(p_{1} u(a)-p_{2} u^{\prime}(a)\right.$ $-A) f(a, u(a), \lambda)<0$ for all $\lambda$ in $\left(u^{\prime}(a), p_{2}^{-1}\left(p_{1} u(a)-A\right)\right]$. Here $u=u_{R}$ or $u_{1}$. Similarly in the case of interior layer behavior we required that if $u\left(t_{0}\right)=\tilde{u}\left(t_{0}\right)=c$ and $\sigma_{L}=u^{\prime}\left(t_{0}^{-}\right) \neq \tilde{u}^{\prime}\left(t_{0}^{+}\right)=\sigma_{R}$ then $\left(\sigma_{R}-\sigma_{L}\right) f\left(t_{0}, c, \lambda\right)>0$ for all $\lambda$ in $\left(\sigma_{L}, \sigma_{R}\right)$. However it is possible that the same qualitative results are valid if these strict inequalities are replaced by suitable nonstrict ones. We term such phenomena "singular" since they invariably involve the case in which the reduced equation $f=0$ is singular at one or more points in $(a, b)$ and along various solution trajectories. For example, if $f_{y^{\prime}}\left(t_{0}, y, y^{\prime}\right)=0$ for all $y, y^{\prime}$ of interest then the point $t_{0}$ is a singular point of $f(\mathrm{cf}$. [10, Chapter 3]). It will become apparent shortly that the assumption of $y$-stability is crucial in obtaining the analogs of the theorems of the previous section. This is not surprising since if $f\left(t_{0}, u\left(t_{0}\right), u^{\prime}\left(t_{0}\right)\right)=0$ and $f_{y^{\prime}}\left(t_{0}, u\left(t_{0}\right), u^{\prime}\left(t_{0}\right)\right)=0$ the solution $u$ loses $y^{\prime}$-stability in passing through $t_{0}$ and so it has to derive stability from the $y$ variable.

Consider first the case of boundary layer behavior. We only state and prove the analog of Theorem 4.1 and then comment on the modifications necessary for proving the analogs of the other boundary layer results.

THEOREM 5.1. Assume that the reduced problem $\left(\Re_{R}\right)$ has a solution $u=u_{R}(t)$ which exists in $[a, b]$ and which is weakly $y^{\prime}$-stable and $y$-stable. Assume also that

$$
\left(p_{1} u_{R}(a)-p_{2} u_{R}^{\prime}(a)-A\right) f\left(t, y, y^{\prime}\right) \leqslant 0 \quad \text { for }\left(t, y, y^{\prime}\right) \text { in } \mathscr{D}\left(u_{R}\right) \cap[a, a+\delta] \text {. }
$$

Then there exists an $\varepsilon_{0}>0$ such that the problem $(\Re)$ with $p_{2}>0$ has a solution $y=y(t, \varepsilon)$ whenever $0<\varepsilon \leqslant \varepsilon_{0}$. In addition, for $t$ in $[a, b]$ we have that

$$
y(t, \varepsilon)=u_{R}(t)+\vartheta\left(w_{L}(t, \varepsilon)\right)+\vartheta(\varepsilon)
$$

and

$$
y^{\prime}(t, \varepsilon)=u_{R}^{\prime}(t)+\vartheta\left(w_{L}^{\prime}(t, \varepsilon)\right)+\vartheta(\varepsilon)
$$

where $w_{L}(t, \varepsilon)=-\left(m^{-1} \varepsilon\right)^{1 / 2} p_{2}^{-1}\left(p_{1} u_{R}(a)-p_{2} u_{R}^{\prime}(a)-A\right) e^{-\left(m e^{-1}\right)^{1 / 2}(t-a)}$ is a solution of $\varepsilon z^{\prime \prime}=m z, a<t<b, z^{\prime}(a, \varepsilon)=p_{2}^{-1}\left(p_{1} u_{R}(a)-p_{2} u_{R}^{\prime}(a)-A\right)$.

Proof. It is only necessary to construct appropriate bounding functions $\alpha$ and $\beta$. Define for $a \leqslant t \leqslant b$ and $\varepsilon>0$,

$$
\left.\begin{array}{l}
\alpha(t, \varepsilon)=u_{R}(t)-\varepsilon \gamma m^{-1} \\
\beta(t, \varepsilon)=u_{R}(t)+w_{L}(t, \varepsilon)+\varepsilon \gamma m^{-1}
\end{array}\right\} \quad \text { if } p_{1} u_{R}(a)-p_{2} u_{R}^{\prime}(a) \leqslant A
$$

and

$$
\left.\begin{array}{l}
\alpha(t, \varepsilon)=u_{R}(t)+w_{L}(t, \varepsilon)-\varepsilon \gamma m^{-1} \\
\beta(t, \varepsilon)=u_{R}(t)+\varepsilon \gamma m^{-1}
\end{array}\right\} \quad \text { if } p_{1} u_{R}(a)-p_{2} u_{R}^{\prime}(a) \geqslant A .
$$

Consider just the case $p_{1} u_{R}(a)-p_{2} u_{R}^{\prime}(a) \leqslant A$. Clearly $\varepsilon \alpha^{\prime \prime} \geqslant f\left(t, \alpha, \alpha^{\prime}\right)$ since $u_{R}$ is $y$-stable. As for $\beta$ we have that

$$
\begin{aligned}
f\left(t, \beta, \beta^{\prime}\right) & =f\left(t, u_{R}, u_{R}^{\prime}\right)+\left(f\left(t, \beta, u_{R}^{\prime}\right)-f\left(t, u_{R}, u_{R}^{\prime}\right)\right)+\left\{f\left(t, \beta, \beta^{\prime}\right)-f\left(t, \beta, u_{R}^{\prime}\right)\right\} \\
& =f_{y}\left(t, \xi, u_{R}^{\prime}\right)\left(\beta-u_{R}\right)+\{\cdot\}
\end{aligned}
$$


where $\xi$ is the appropriate intermediate point. Therefore,

$$
\begin{aligned}
f\left(t, \beta, \beta^{\prime}\right)-\varepsilon \beta^{\prime \prime} & =f_{y}\left(t, \xi, u_{R}^{\prime}\right)\left(w_{L}+\varepsilon \gamma m^{-1}\right)+\{\cdot\}-\varepsilon u_{R}^{\prime \prime}-\varepsilon w_{L}^{\prime \prime} \\
& \geqslant m w_{L}+\varepsilon \gamma-\{\cdot\}-\varepsilon M-\varepsilon w_{L}^{\prime \prime} \quad\left(M=\max \left|u_{R}^{\prime \prime}\right|\right) \\
& \geqslant \varepsilon \gamma-\varepsilon M+\{\cdot\}
\end{aligned}
$$

by our choice of $w_{L}$. Now for $t$ in $[a, a+\delta]$ the expression in parentheses is nonnegative by assumption and for $t$ in $[a+\delta, b]$ it is transcendentally small, that is, $\{\cdot\}=\mathcal{O}\left(\varepsilon^{N}\right)$ for all $N \geqslant 1$. Consequently, if $\gamma=M+1$ and $\varepsilon$ is sufficiently small, say $0<\varepsilon \leqslant \varepsilon_{0}$, we have the desired inequality $\varepsilon \beta^{\prime \prime} \leqslant f\left(t, \beta, \beta^{\prime}\right)$ in $(a, b)$.

For the situations described in Theorems 4.2 and 4.5 the corresponding "singular" analogs are valid if the locally weakly $y^{\prime}$-stable function $u_{I}$ satisfies (5.1) with $u_{R}$ replaced by $u_{I}$ and if

$$
\left(q_{1} u_{I}(b)+q_{2} u_{I}^{\prime}(b)-B\right) f\left(t, y, y^{\prime}\right) \leqslant 0 \quad \text { for }\left(t, y, y^{\prime}\right) \text { in } \mathscr{D}\left(u_{I}\right) \cap[b-\delta, b] .
$$

Consider next the case of interior layer behavior. Once again we will only state and prove the analog of Theorem 4.3 and simply indicate the modifications required in the other interior layer results.

THEOREM 5.2. Assume that the reduced problems $\left(\Re_{L}\right)$ and $\left(\Re_{R}\right)$ have solutions $u=u_{L}(t)$ and $u=u_{R}(t)$ in $\left[a, t_{L}\right)$ and $\left(t_{R}, b\right]$ respectively with $t_{R}<t_{L}$ such that $u_{L}\left(t_{0}\right)=u_{R}\left(t_{0}\right)$ and $\sigma_{L}=u_{L}^{\prime}\left(t_{0}\right) \neq u_{R}^{\prime}\left(t_{0}\right)=\sigma_{R}$ at a point $t_{0}$ in $\left(t_{R}, t_{L}\right)$. Assume also that the path $u=u_{1}(t)$ is weakly $y^{\prime}$-stable and $y$-stable, and that

$$
\left(\sigma_{R}-\sigma_{L}\right) f\left(t, y, y^{\prime}\right) \geqslant 0 \text { for }\left(t, y, y^{\prime}\right) \text { in } \mathscr{D}\left(u_{1}\right) \cap\left[t_{0}-\delta, t_{0}+\delta\right] \text {. }
$$

Then there exists an $\varepsilon_{0}>0$ such that the problem $(\mathcal{U})$ has a solution $y=y(t, \varepsilon)$ whenever $0<\varepsilon \leqslant \varepsilon_{0}$. In addition, we have that

$$
\begin{gathered}
y(t, \varepsilon)=u_{1}(t)+\vartheta\left(w_{l}(t, \varepsilon)\right)+\vartheta\left(w_{r}(t, \varepsilon)\right)+\vartheta(\varepsilon) \text { for } a \leqslant t \leqslant b, \\
y^{\prime}(t, \varepsilon)=u_{L}^{\prime}(t)+\vartheta\left(w_{l}^{\prime}(t, \varepsilon)\right)+\vartheta(\varepsilon) \text { for } a \leqslant t \leqslant t_{0}
\end{gathered}
$$

and

$$
y^{\prime}(t, \varepsilon)=u_{R}^{\prime}(t)+\vartheta\left(w_{r}^{\prime}(t, \varepsilon)\right)+\vartheta(\varepsilon) \text { for } t_{0} \leqslant t<b .
$$

Here $w_{l}(t, \varepsilon)=\frac{1}{2}\left(\varepsilon m^{-1}\right)^{1 / 2}\left(\sigma_{R}-\sigma_{L}\right) e^{\left(\varepsilon^{-1} m\right)^{1 / 2}\left(t-t_{0}\right)}$ is a solution of $\varepsilon z^{\prime \prime}=m z, a<t<$ $t_{0}, \quad z^{\prime}\left(t_{0}^{-}, \varepsilon\right)=\frac{1}{2}\left(\sigma_{R}-\sigma_{L}\right)$, and $w_{r}(t, \varepsilon)=\frac{1}{2}\left(\varepsilon m^{-1}\right)^{1 / 2}\left(\sigma_{R}-\sigma_{L}\right) e^{-\left(\varepsilon^{-1} m\right)^{1 / 2}\left(t-t_{0}\right)}$ is a solution of $\varepsilon z^{\prime \prime}=m z, t_{0}<t<b, z^{\prime}\left(t_{0}^{+}, \varepsilon\right)=\frac{1}{2}\left(\sigma_{L}-\sigma_{R}\right)$.

Proof. Suppose for example that $\sigma_{L}<\sigma_{R}$ and define for $\varepsilon>0$,

$$
\alpha(t, \varepsilon)=u_{1}(t)-\varepsilon \gamma m^{-1}, \quad a \leqslant t \leqslant b,
$$

and

$$
\beta(t, \varepsilon)= \begin{cases}u_{L}(t)+w_{l}(t, \varepsilon)+\varepsilon \gamma m^{-1}, & a \leqslant t \leqslant t_{0}, \\ u_{R}(t)+w_{r}(t, \varepsilon)+\varepsilon \gamma m^{-1}, & t_{0} \leqslant t \leqslant b .\end{cases}
$$

Then it is a straightforward matter to show that for $\gamma$ sufficiently large and $\varepsilon$ sufficiently small, say $0<\varepsilon \leqslant \varepsilon_{0}$, these functions satisfy the correct inequalities. 
Regarding the analogs of Theorems 4.4 and 4.5 we must assume that

$$
\left(\mu_{j}-\sigma_{j}\right) f\left(t, y, y^{\prime}\right) \geqslant 0 \text { for }\left(t, y, y^{\prime}\right) \text { in } \mathscr{D}(u) \cap\left[t_{j}-\delta, t_{j}+\delta\right] \text {. }
$$

Here $j=1$ and /or 2 and $u=u_{2}$ or $u_{3}$.

We close this section with two remarks.

REMARK 5.1. In discussing certain problems it is necessary to amend condition (5.1) as follows. First of all, if $u_{R}^{\prime \prime} \geqslant 0(\leqslant 0)$ in $[a, a+\delta]$ and $p_{1} u_{R}(a)-p_{2} u_{R}^{\prime}(a)<$ $A(>A)$ then the proof of Theorem 5.1 shows that it is enough to assume in place in $(5.1)$ that

$$
f\left(t, u_{R}(t), \lambda\right) \geqslant 0(\leqslant 0) \text { for } t \text { in }[a, a+\delta]
$$

and for all $\lambda$ in $\left[p_{2}^{-1}\left(p_{1} u_{R}(a)-A\right), u_{R}^{\prime}(a)\right)\left(\left(u_{R}^{\prime}(a), p_{2}^{-1}\left(p_{1} u_{R}(a)-A\right)\right]\right)$. Secondly, if in the original condition (5.1), $\left(p_{1} u_{R}(a)-p_{2} u_{R}^{\prime}(a)-A\right) f\left(t, y, y^{\prime}\right)<\mu(\varepsilon)$ for a positive function $\mu$ which is such that $\mu(\varepsilon) \leqslant L(\varepsilon) \varepsilon$ with $L(\varepsilon)=\mathcal{O}(1)$ depending only on $\varepsilon$, then the conclusion of Theorem 5.1 remains valid. Similar remarks apply to the condition (5.2).

It is of ten necessary to amend also the condition (5.3). If $u_{L}^{\prime \prime}>0(<0)$ and $u_{R}^{\prime \prime} \geqslant 0(\leqslant 0)$ in $\left[t_{0}-\delta, t_{0}\right]$ and $\left[t_{0}, t_{0}+\delta\right]$ respectively and if $\sigma_{L}<\sigma_{R}\left(\sigma_{L}>\sigma_{R}\right)$ then the conclusion of Theorem 5.2 is valid if (5.3) is replaced by

$$
\begin{aligned}
f\left(t, u_{1}(t), \lambda\right) \geqslant 0(\leqslant 0) \text { for } t \text { in }\left[t_{0}-\delta, t_{0}+\delta\right] \text { and } \\
\\
\text { for all } \lambda \text { in }\left(\sigma_{L}, \sigma_{R}\right)\left(\left(\sigma_{R}, \sigma_{L}\right)\right) .
\end{aligned}
$$

Secondly, if in (5.3) $\left(\sigma_{R}-\sigma_{L}\right) f\left(t, y, y^{\prime}\right) \leqslant \mu(\varepsilon)$ with $\mu$ as before then the conclusion of Theorem 5.2 is also valid. The conditions (5.4) can be modified in a similar manner.

REMARK 5.2. If we assume in the theorems of this section that the reduced solutions are $y$-stable in a sense more general than that given in Definition 4.6 then the layer corrector terms $w$ must be modified accordingly (cf. for example [9]). The qualitative results are nevertheless the same.

6. Some examples. We discuss now several examples that illustrate the theory in $\S \S 4$ and 5.

EXAmPle 6.1. Consider the problem

$$
\begin{aligned}
\varepsilon y^{\prime \prime}=y-t y^{\prime}-y^{\prime 3} & =f\left(t, y, y^{\prime}\right), \quad-1<t<1, \\
-y^{\prime}(-1, \varepsilon) & =A, \quad y(1, \varepsilon)=B .
\end{aligned}
$$

Note that solutions of (E4) are unique by the maximum principle (cf. [16]). The reduced equation $u=t u^{\prime}+u^{\prime 3}$ is a Clairaut equation (cf. [10, Chapter 3]) whose solutions are the straight lines $u=u(t)=c t+c^{3}$ and their envelope $u=$ $\pm 2(-t)^{3 / 2} / 3 \sqrt{3}$ which is a singular solution defined for $t<0$ (see Figure 1).

Suppose first that $B=2$. Then the straight line $u=u_{R}(t)=t+1$ is a solution of the reduced problem $\left(\Re_{R}\right)$ corresponding to (E4) which is strongly $y^{\prime}$-stable in $[-1,1]$ since $f_{y}\left[u_{R}(t)\right]=-t-3 \leqslant-2$ there. In order to apply Theorem 4.1 we must determine for what values of $A$

$$
\left(-u_{R}^{\prime}(-1)-A\right) f\left(-1, u_{R}(-1), \lambda\right)=(-1-A) \lambda\left(1-\lambda^{2}\right)<0
$$




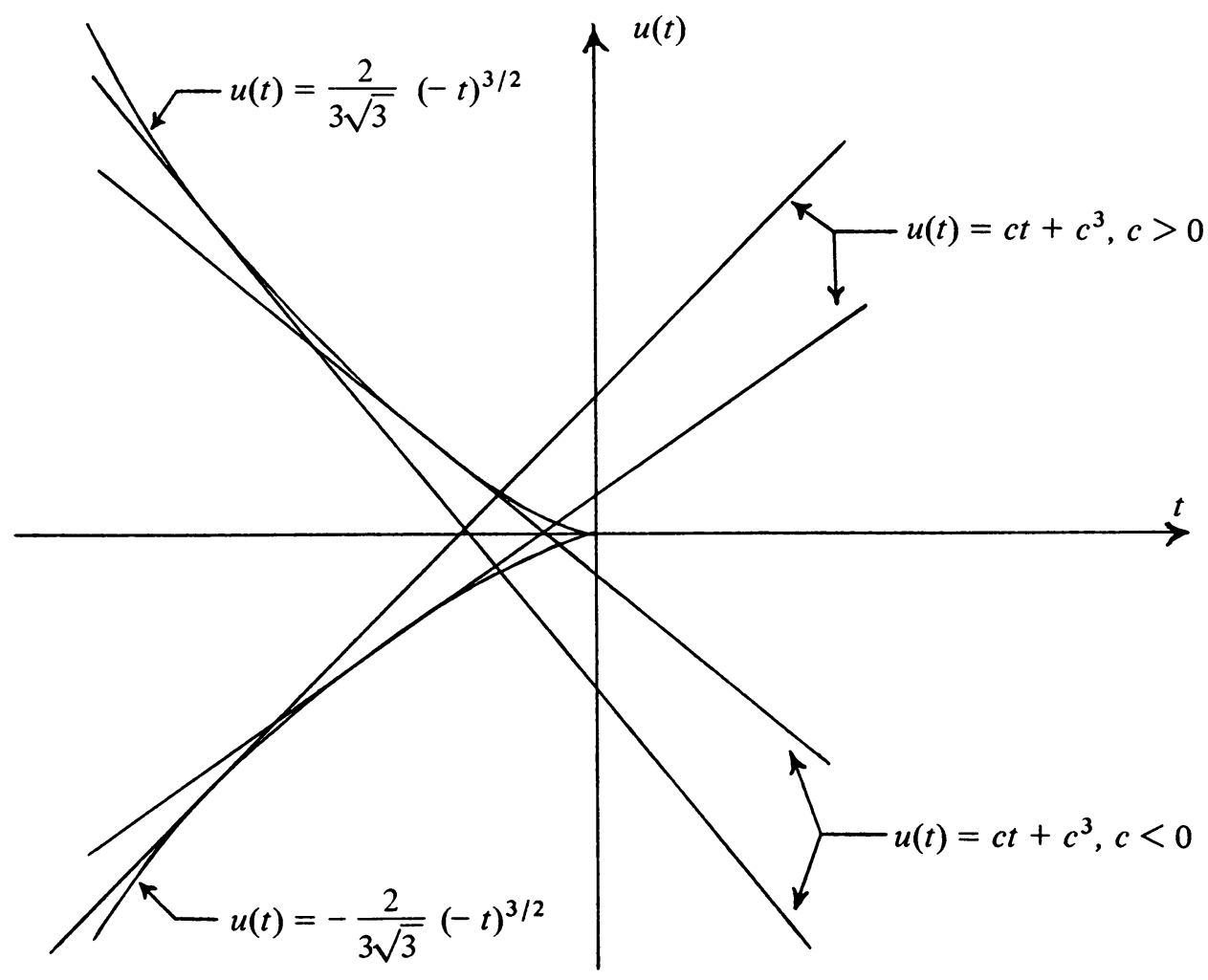

Figure 1

Solutions of the Clairaut equation $u=t u^{\prime}+u^{\prime 3}$

for all $\lambda$ in $\left(u_{R}^{\prime}(-1)=1,-A\right]$ or $[-A, 1)$. If $A=-1$ then $y(t, \varepsilon)=u_{R}(t)$ is the solution of (E4). If $A>-1$ then $(-1-A) \lambda\left(1-\lambda^{2}\right)<0$ for $\lambda$ in $[-A, 1)$ provided $-A>0$, that is, for $A<0$. Similarly, if $A<-1$ then

$$
(-1-A) \lambda\left(1-\lambda^{2}\right)<0
$$

for $\lambda$ in $(1,-A]$ provided $-A>1$, that is, for $A<-1$. Thus by Theorem 4.1 the problem (E4) for $A<0$ has a solution $y=y(t, \varepsilon)$ such that $y(t, \varepsilon) \rightarrow u_{R}(t)=t+1$ in $[-1,1]$ and $y^{\prime}(t, \varepsilon) \rightarrow 1$ in $(-1,1]$ as $\varepsilon \rightarrow 0^{+}$.

Suppose next that $A=0$ and $B=5 / 8$. Then $u=u_{L} \equiv 0$ is a solution of the reduced problem $\left(\Re_{L}\right)$ and $u=u_{R}(t)=\frac{1}{2} t+\frac{1}{8}$ is a solution of $\left(\Re_{R}\right)$ which intersect at $t_{0}=-\frac{1}{4}$. The corresponding angular path $u=u_{1}(t)$ is strongly $y^{\prime}$-stable since $f_{y}\left[u_{L}(t)\right]=-t \geqslant \frac{1}{4}$ in $\left[-1,-\frac{1}{4}\right]$ and $f_{y}\left[u_{R}(t)\right]=-t-\frac{3}{4}<-\frac{1}{2}$ in $\left[-\frac{1}{4}, 1\right]$. To apply Theorem 4.3 we must check the condition that

$$
\left(\sigma_{R}-\sigma_{L}\right) f\left(-\frac{1}{4}, 0, \lambda\right)>0
$$

for $\lambda$ between $\sigma_{L}$ and $\sigma_{R}$, that is,

$$
\frac{1}{2} f\left(-\frac{1}{4}, 0, \lambda\right)=\frac{1}{2} \lambda\left(\frac{1}{4}-\lambda^{2}\right)>0
$$


for $\lambda$ in $\left(0, \frac{1}{2}\right)$. Thus we deduce from Theorem 4.3 that the problem (E4) has a solution $y=y(t, \varepsilon)$ such that

$$
y(t, \varepsilon) \rightarrow u_{1}(t)=\left\{\begin{array}{l}
0, \quad-1 \leqslant t \leqslant-\frac{1}{4}, \\
\frac{1}{2} t+\frac{1}{8}, \quad-\frac{1}{4} \leqslant t \leqslant 1,
\end{array}\right.
$$

and

$$
y^{\prime}(t, \varepsilon) \rightarrow \begin{cases}0, & -1 \leqslant t<-\frac{1}{4} \\ \frac{1}{2}, & -\frac{1}{4}<t \leqslant 1\end{cases}
$$

as $\varepsilon \rightarrow 0^{+}$.

We consider finally two applications of Theorem 4.5. Set $B=10 / 27$. The unique solution $u$ of the reduced equation $f=0$ satisfying $u(1)=B$ is $u=u_{R}(t)=t / 3+$ $1 / 27$ and it intersects the lower branch $u_{I}\left(=-2(-t)^{3 / 2} / 3 \sqrt{3}\right)$ of the singular solution at the point $t_{2}=-1 / 3$ (cf. Figure 1). Since $u_{I}$ is singular we also know that $u_{I}^{\prime}\left(t_{2}\right)=u_{R}^{\prime}\left(t_{2}\right)$ and so the corresponding reduced path $u=u_{3}(t)$ is of class $C^{(1)}[-1,1]$. It remains for us to determine the values of $A$ for which

$$
\left(-u_{I}^{\prime}(-1)-A\right) f\left(-1, u_{I}(-1), \lambda\right)=(-1 / \sqrt{3}-A)\left(-2 / 3 \sqrt{3}+\lambda-\lambda^{3}\right)<0
$$

for $\lambda$ in $(1 / \sqrt{3},-A]$ or $[-A, 1 / \sqrt{3})$. Note that if $A=-1 / \sqrt{3}$ then $y(t, \varepsilon) \rightarrow$ $u_{3}(t)$ and $y^{\prime}(t, \varepsilon) \rightarrow u_{3}^{\prime}(t)$ in $[-1,1]$ as $\varepsilon \rightarrow 0^{+}$. If however $-1 / \sqrt{3}-A>0$ then

$$
\begin{aligned}
(-1 / \sqrt{3}-A)( & \left.-2 / 3 \sqrt{3}+\lambda-\lambda^{3}\right) \\
= & (1 / \sqrt{3}+A)(\lambda-1 / \sqrt{3})^{2}(\lambda+2 / \sqrt{3})<0
\end{aligned}
$$

for all $\lambda$ in $(1 / \sqrt{3},-A]$, while if $-1 / \sqrt{3}-A<0$ then

$$
(1 / \sqrt{3}+A)(\lambda-1 / \sqrt{3})^{2}(\lambda+2 / \sqrt{3}) \Varangle 0
$$

for $\lambda$ in $[-2 / \sqrt{3}, 1 / \sqrt{3})$. Consequently we can apply Theorem 4.5 only if $A \leqslant-1 / \sqrt{3}$ to conclude that the problem (E4) has a solution $y=y(t, \varepsilon)$ such that $y(t, \varepsilon) \rightarrow u_{3}(t)$ in $[-1,1]$ and $y^{\prime}(t, \varepsilon) \rightarrow u_{3}^{\prime}(t)$ in $(-1,1]$ as $\varepsilon \rightarrow 0^{+}$.

Suppose finally that $A=1 / 3$ and $B=26 / 27$. Then the unique solution $u$ of $f=0$ satisfying $u(1)=B$ is $u=u_{R}(t)=2 t / 3+8 / 27$ and it intersects the upper branch $u_{I}\left(=2(-t)^{3 / 2} / 3 \sqrt{3}\right)$ of the singular solution at $t_{2}=-1 / 3$. For this choice of $A$ and $B$ note that $\sigma_{L}=u_{I}^{\prime}(-1 / 3)=-1 / 3<u_{R}^{\prime}(-1 / 3)=2 / 3=\sigma_{R}$ in contrast to the previous problem. We first check the condition for a crossing at $t_{2}$, namely

$$
\left(\sigma_{R}-\sigma_{L}\right) f\left(-\frac{1}{3}, \frac{2}{27}, \lambda\right)=\frac{2}{27}+\frac{1}{3} \lambda-\lambda^{3}>0
$$

for $\lambda$ in $\left(-\frac{1}{3}, \frac{2}{3}\right)$. But

$$
-\lambda^{3}+\frac{1}{3} \lambda+\frac{2}{27}=-\left(\lambda+\frac{1}{\sqrt{3}}\right)^{2}\left(\lambda-\frac{2}{3}\right)>0
$$


for such $\lambda$ and so there is a crossing at $t_{2}$. Thus Theorem 4.5 tells us that there is a solution $y=y(t, \varepsilon)$ of (E4) such that

$$
y(t, \varepsilon) \rightarrow u_{2}(t)= \begin{cases}u_{I}(t), & -1 \leqslant t \leqslant-\frac{1}{3}, \\ u_{R}(t), & -\frac{1}{3} \leqslant t \leqslant 1,\end{cases}
$$

and

$$
y^{\prime}(t, \varepsilon) \rightarrow \begin{cases}u_{I}^{\prime}(t), & -1 \leqslant t<-\frac{1}{3}, \\ u_{R}^{\prime}(t), & -\frac{1}{3}<t \leqslant 1\end{cases}
$$

as $\varepsilon \rightarrow 0^{+}$.

EXAMPLE 6.2. Consider now the problem

$$
\begin{aligned}
& \varepsilon y^{\prime \prime}=y+t y^{\prime}+y^{\prime n}=f\left(t, y, y^{\prime}\right), \quad-1<t<1, \\
& y(-1, \varepsilon)-y^{\prime}(-1, \varepsilon)=A, \quad y(1, \varepsilon)+y^{\prime}(1, \varepsilon)=B,
\end{aligned}
$$

for $n$ an integer greater than two, which we will use to illustrate Theorem 4.2. Once again solutions of (E5) are unique by the maximum principle. The function $u_{I} \equiv 0$ is clearly a solution of the reduced equation $f=0$ which is locally strongly $y^{\prime}$-stable since $f_{y},[0]=t$. Suppose first that $n$ is odd. In order to apply Theorem 4.2 we must consider inequalities at $t=-1$ and $t=1$, namely

$$
\begin{array}{cc}
\left(u_{I}(-1)-u_{I}^{\prime}(-1)-A\right) f\left(-1, u_{I}(-1), \lambda\right)<0 & \text { for } \lambda \text { in }\left(u_{I}^{\prime}(-1), u_{I}(-1)-A\right] \\
& \text { or }\left[u_{I}(-1)-A, u_{I}^{\prime}(-1)\right)
\end{array}
$$

and

$$
\begin{gathered}
\left(u_{I}(1)+u_{I}^{\prime}(1)-B\right) f\left(1, u_{I}(1), \lambda\right)<0 \quad \text { for } \lambda \text { in }\left(u_{I}^{\prime}(1), B-u_{I}(1)\right] \\
\text { or }\left[B-u_{I}(1), u_{I}^{\prime}(1)\right) .
\end{gathered}
$$

Condition (6.1) is equivalent to $-A \lambda\left(1-\lambda^{n-1}\right)<0$ for $\lambda$ in $(0,-A]$ or $[-A, 0)$ and this is satisfied for $|A|<1(A \neq 0)$ since $n$ is odd. On the other hand, condition (6.2) is equivalent to $-B \lambda\left(1+\lambda^{n-1}\right)<0$ for $\lambda$ in $(0, B]$ or $[B, 0)$, which is true for all $B \neq 0$. If $A=B=0$ then $y(t, \varepsilon) \equiv 0$ is the solution of (E5) and so from Theorem 4.2 we deduce that if $n$ is odd and $|A|<1$ then for all values of $B$ the problem (E5) has a solution $y=y(t, \varepsilon)$ such that

$$
y(t, \varepsilon) \rightarrow 0 \text { in }[-1,1] \text { and } y^{\prime}(t, \varepsilon) \rightarrow 0 \text { in }(-1,1) \text { as } \varepsilon \rightarrow 0^{+} \text {. }
$$

If now $n$ is even then condition (6.1) is clearly satisfied by all values of $A>-1$ $(A \neq 0)$ while condition (6.2) is satisfied by all values of $B>-1(B \neq 0)$. Thus from Theorem 4.2 we deduce that if $n$ is even and $A, B>-1$ then the problem (E5) has a solution $y=y(t, \varepsilon)$ satisfying the limiting relations (6.3).

EXAMPLE 6.3. Consider next the problem

$$
\begin{aligned}
& \varepsilon y^{\prime \prime}=t y^{\prime 3}+y^{3}-y=f\left(t, y, y^{\prime}\right), \quad-1<t<1, \\
& y(-1, \varepsilon)-y^{\prime}(-1, \varepsilon)=A, \quad y(1, \varepsilon)+y^{\prime}(1, \varepsilon)=B .
\end{aligned}
$$

We will show that for certain choices of $A$ and $B$ this problem has at least two solutions. 
The reduced equation $f=0$ has many solutions but we single out just the constant ones $u_{1} \equiv 1$ and $u_{2} \equiv-1$ which are $y$-stable since $f_{y}[ \pm 1]=2$. Note also that both $u_{1}$ and $u_{2}$ are locally weakly $y^{\prime}$-stable since $f_{y^{\prime}}=3 t y^{\prime 2}$. We consider only $u_{1}$ in detail since the corresponding results for $u_{2}$ follow by reflection $(y \rightarrow-y)$. To apply Theorem 4.2 we must check the two inequalities:

$$
\begin{array}{cc}
\left(u_{1}(-1)-u_{1}^{\prime}(-1)-A\right) f\left(-1, u_{1}(-1), \lambda\right)<0 & \text { for } \lambda \text { in }\left(u_{1}^{\prime}(-1), u_{1}(-1)-A\right] \\
& \text { or }\left[u_{1}(-1)-A, u_{1}^{\prime}(-1)\right) ; \\
\left(u_{1}(1)+u_{1}^{\prime}(1)-B\right) f\left(1, u_{1}(1), \lambda\right)<0 & \text { for } \lambda \text { in }\left(u_{1}^{\prime}(1), B-u_{1}(1)\right] \\
& \text { or }\left[B-u_{1}(1), u_{1}^{\prime}(1)\right) .
\end{array}
$$

Condition (6.4) is equivalent to $(1-A) \lambda^{3}>0$ for $\lambda$ in $(0,1-A]$ or $[1-A, 0)$, which is satisfied for all $A \neq 1$. Similarly condition $(6.5)$ is equivalent to $(1-B) \lambda^{3}$ $<0$ for $\lambda$ in $(0, B-1]$ or $[B-1,0)$, which is satisfied for all $B \neq 1$. Thus by Theorem 4.2 the problem (E6) has a solution $y=y_{1}(t, \varepsilon)$ such that for all $A$ and $B$, $y_{1}(t, \varepsilon) \rightarrow 1$ in $[-1,1]$ and $y_{1}^{\prime}(t, \varepsilon) \rightarrow 0$ in $(-1,1)$ as $\varepsilon \rightarrow 0^{+}$. Consequently this problem has another solution $y=y_{2}(t, \varepsilon)$ such that for all $A$ and $B y_{2}(t, \varepsilon) \rightarrow-1$ in $[-1,1]$ and $y_{2}^{\prime}(t, \varepsilon) \rightarrow 0$ in $(-1,1)$ as $\varepsilon \rightarrow 0^{+}$.

EXAMPLE 6.4. In this final example we illustrate some of the singular phenomena discussed in $\$ 5$. The problem is

$$
\begin{gathered}
\varepsilon y^{\prime \prime}=y-t y^{\prime 3}=f\left(t, y, y^{\prime}\right), \quad a<t<b, \\
-y^{\prime}(a, \varepsilon)=A, \quad q_{1} y(b, \varepsilon)+q_{2} y^{\prime}(b, \varepsilon)=B,
\end{gathered}
$$

whose solutions are unique by the maximum principle. Suppose first that $a=q_{2}=$ $B=0$ and $b=q_{1}=1$, and consider the function $u=u_{R}(t) \equiv 0$. Clearly $u_{R}$ is a solution of $f=0$ satisfying $u_{R}(1)=B$ which is locally weakly $y^{\prime}$-stable since $f_{y^{\prime}}=-3 t y^{\prime 2}$. Now $f(0,0,0)=0$ and so we cannot apply Theorem 4.1 but we suspect that for all values of $A$ there is a solution $y=y(t, \varepsilon)$ of $(\mathrm{E} 7)$ such that

$$
y(t, \varepsilon) \rightarrow 0 \text { in }[0,1] \text { and } y^{\prime}(t, \varepsilon) \rightarrow 0 \text { in }(0,1] \text { as } \varepsilon \rightarrow 0^{+} \text {. }
$$

To establish this we note that condition (5.1)' (cf. Remark 5.1) of Theorem 5.1 holds, namely

$$
\left(-u_{R}^{\prime}(0)-A\right) f\left(t, u_{R}(t), \lambda\right)=A t \lambda^{3} \leqslant 0
$$

for $t$ in $[0, \delta]$ and $\lambda$ in $(0,-A]$ or $[-A, 0)$. Thus by Theorem 5.1 the problem (E7) has a solution $y=y(t, \varepsilon)$ satisfying the limiting relations (6.6) for all values of $A$.

Suppose next that $a=-1, A=q_{1}=q_{2}=1$ and $B=2$, and consider the functions $u=u_{L}(t)=-t$ and $u=u_{R}(t)=t$. Clearly $u_{L}$ is a solution of the corresponding reduced problem $\left(\Re_{L}\right)$ while $u_{R}$ is a solution of $\left(\Re_{R}\right)$. These functions intersect at $t_{0}=0$ and the angular path $u=u_{1}(t)=|t|$ is weakly $y^{\prime}$-stable since $f_{y^{\prime}}=-3 t y^{\prime 2}$. However Theorem 4.3 is inapplicable because $f(0,0, \lambda) \equiv 0$ for all $\lambda$. We are led to consider applying Theorem 5.2 since $u_{1}$ is $y$-stable and so we have to verify condition (5.3)' (cf. Remark 5.1), that is, $f\left(t, u_{1}(t), \lambda\right) \geqslant 0$ for $|t| \leqslant \delta$ and $|\lambda|<1$. For $t$ in $[-\delta, 0], f\left(t, u_{1}(t), \lambda\right)=-t\left(1+\lambda^{3}\right) \geqslant 0$ and for $t$ in $[0, \delta], f\left(t, u_{1}(t), \lambda\right)=$ $t\left(1-\lambda^{3}\right) \geqslant 0$. Therefore Theorem 5.2 tells us that the problem (E7) has a solution 
$y=y(t, \varepsilon)$ such that $y(t, \varepsilon) \rightarrow|t|$ in $[-1,1]$ and

$$
y^{\prime}(t, \varepsilon) \rightarrow\left\{\begin{array}{l}
-1, \quad-1 \leqslant t<0, \\
1, \quad 0<t \leqslant 1,
\end{array}\right.
$$

as $\varepsilon \rightarrow 0^{+}$.

7. Concluding remarks. We close with some observations on the assumptions and the conclusions of the theorems presented here.

First of all, in the case of more widely studied singularly perturbed boundary value problems, for instance quasilinear Dirichlet problems (that is, $f\left(t, y, y^{\prime}\right)=$ $\vartheta\left(\left|y^{\prime}\right|\right)$ as $\left.\left|y^{\prime}\right| \rightarrow \infty\right)$, one expects both the solution and its first derivative to behave nonuniformly at one or more points in the interval under consideration. Thus the reader may feel that our results here for the Robin problem $(\mathcal{H})$ are incomplete in that a solution always behaves uniformly in $[a, b]$. It is however the assumption that $f$, as a function of $y^{\prime}$, grows faster than $y^{\prime 2}$ which precludes (in most cases) the occurrence of the "usual" types of boundary and interior (shock) layer behavior. This remark, which is not widely known, is due essentially to Vishik and Liusternik, and the reader should consult [17, Chapter II] for a detailed exposition of their results.

Finally, the definitions of stability which we have used to derive our results may appear somewhat mysterious or artificial at first glance, especially those involving local $y^{\prime}$-stability and/or $y$-stability. With regard to $y^{\prime}$-stability we were led to make such definitions based on the observation that in order for boundary layer behavior to occur at $t=a(t=b)$ the linearized coefficient of $y^{\prime}$ must be nonpositive (nonnegative). Here we linearize about the appropriate reduced path. The definition of $y^{\prime}$-stability for an angular path then becomes more natural if we regard each angular point as a "two-sided" boundary point. With regard to $y$-stability we observed first that under such an assumption a (local) maximum principle applied (cf. [16, Chapter 1]) and so solutions of $(\mathscr{N})$ satisfy a priori bounds which are independent of $\varepsilon$. Moreover, at points of nonuniformity where $f_{y^{\prime}}$ vanishes the layer corrector terms are solutions of the equation $\varepsilon z^{\prime \prime}=m z$, where $m>0$ is the lower bound on $f_{y}$. Thus it seemed natural to us to impose such a restriction, especially when the absence of $y$-stability (even in the linear case) can lead to formidable difficulties involving resonant behavior of solutions.

ACKNOWLedgement. Portions of this paper were written at the University of Southern California and the University of Arizona and the author wishes to thank Professors W. A. Harris, Jr. and R. E. O'Malley, Jr. for their hospitality. He also wishes to thank the NSF and the ONR for their financial support.

\section{REFERENCES}

1. E. A. Coddington and N. Levinson, $A$ boundary value problem for a nonlinear differential equation with a small parameter, Proc. Amer. Math. Soc. 3 (1952), 73-81.

2. F. W. Dorr, S. V. Parter and L. F. Shampine, Applications of the maximum principle to singular perturbation problems, SIAM Rev. 15 (1973), 43-88.

3. L. E. El'sgol'c, Qualitative methods in mathematical analysis, Transl. Math. Mono., No. 12, Amer. Math. Soc., Providence, R. I., 1964. 
4. A. Erdélyi, Singular perturbations of boundary value problems involving ordinary differential equations, SIAM J. Appl. Math. 11 (1963), 105-116.

5. S. Haber and N. Levinson, $A$ boundary value problem for a singularly perturbed differential equation, Proc. Amer. Math. Soc. 6 (1955), 866-872.

6. W. Hahn, Stability of motion, Die Grundlehren der mathematischen Wissenschaften, SpringerVerlag, New York, 1967.

7. J. W. Heidel, A second-order nonlinear boundary value problem, J. Math. Anal. Appl. 48 (1974), 493-503.

8. F. A. Howes, An asymptotic theory for a class of nonlinear Robin problems, J. Differential Equations 30 (1978), 192-234.

9. Singularly perturbed superquadratic boundary value problems, J. Nonlinear Analysis 3 (1979), 175-192.

10. E. L. Ince, Ordinary differential equations, Dover, New York, 1956.

11. L. K. Jackson, Subfunctions and second-order ordinary differential inequalities, Advances in Math. 2 (1968), 307-363.

12. W. E. Johnson and L. M. Perko, Interior and exterior boundary value problems from the theory of the capillary tube, Arch. Rational Mech. Anal. 29 (1968), 125-143.

13. R. E. O'Malley, Jr., On singular perturbation problems with interior nonuniformities, J. Math. Mech. 19 (1970), 1103-1112.

14. L. M. Perko, Boundary layer analysis of the wide capillary tube, Arch. Rational Mech. Anal. 45 (1972), 120-133.

15. __ Singularly perturbed two-point boundary value problems with $\left(y^{\prime}\right)^{n}, n>2$, nonlinearities, Arch. Rational Mech. Anal. (to appear).

16. M. H. Protter and H. F. Weinberger, Maximum principles in differential equations, Prentice-Hall, Englewood Cliffs, N. J., 1967.

17. A. B. Vasil'eva, Asymptotic behavior of solutions to certain problems involving nonlinear differential equations containing a small parameter multiplying the highest derivatives, Russian Math. Surveys 18 (1963), 13-84.

Department of Mathematics, University of California, Davis, California 95616 